The Astrophysical Journal, submitted

Preprint typeset using LATEX style emulateapj v. 25/04/01

\title{
THE ANISOTROPIC DISTRIBUTION OF GALACTIC SATELLITES
}

\author{
Andrew R. Zentner ${ }^{1}$, Andrey V. Kravtsov ${ }^{1,2}$, Oleg Y. Gnedin ${ }^{3}$, AnAtoly A. Klypin ${ }^{4}$ \\ The Astrophysical Journal, submitted
}

\begin{abstract}
We present a study of the spatial distribution of dwarf satellites (or subhalos) in galactic dark matter halos using dissipationless cosmological simulations of the concordance flat Cold Dark Matter (CDM) model with vacuum energy. We find that subhalos are distributed anisotropically and are preferentially located along the major axes of the triaxial mass distributions of their hosts. The Kolmogorov-Smirnov probability for drawing our simulated subhalo sample from an isotropic distribution is $P_{\mathrm{KS}} \simeq 1.5 \times 10^{-4}$. An isotropic distribution of subhalos is thus not the correct null hypothesis for testing the CDM paradigm. The nearly planar distribution of observed Milky Way $(\mathrm{MW})$ satellites is marginally consistent (probability $\simeq 0.02$ ) with being drawn randomly from the subhalo distribution in our simulations. Furthermore, if we select the subhalos likely to be luminous, we find a distribution that is consistent with the observed MW satellites. In fact, we show that subsamples of the subhalo population with a centrally-concentrated radial distribution that is similar to that of the MW dwarfs typically exhibit a comparable degree of planarity. We explore the origin of the observed subhalo anisotropy and conclude that it is likely due to (1) the preferential accretion of satellites along filaments, often closely aligned with the major axis of the host halo, and (2) evolution of satellite orbits within the prolate, triaxial potentials typical of CDM halos. Agreement between predictions and observations requires the major axis of the outer dark matter halo of the Milky Way to be nearly perpendicular to the disk. We discuss possible observational tests of such disk-halo alignment with current large galaxy surveys.
\end{abstract}

Subject headings: cosmology: theory, large-scale structure of universe - dark matter - galaxies: formation, halos, structure - methods: numerical

\section{INTRODUCTION}

Simulations of structure formation in the standard cold dark matter (CDM) scenario (e.g., Blumenthal et al. 1984) show that virialized dark matter halos teem with distinct, gravitationallybound substructures, often referred to as subhalos. The abundance of subhalos in Milky Way-sized halos has received much attention, as there are more than an order of magnitude fewer observed dwarf satellite galaxies around these systems than the predicted number of subhalos of comparable velocity dispersion (Kauffmann et al. 1993; Klypin et al. 1999a; Moore et al. 1999). This problem is often referred to as the "the missing satellites problem."

The difference in predicted and observed abundances of dwarf satellites is likely related to the corresponding difference in spatial distributions. The predicted radial distribution of subhalo populations is considerably more extended than that of the Milky Way (MW) satellites (Taylor et al. 2003; Kravtsov et al. 2004). This may indicate that the environments in which low-luminosity galaxies form are non-trivially biased relative to the overall population of subhalos. In addition, the dwarf satellites of the MW and M31 appear to be distributed anisotropically about their hosts (e.g., Lynden-Bell 1982; Majewski 1994; Hartwick 1996, 2000; Mateo 1998; Grebel et al. 1999; Willman et al. 2004). Kroupa et al. (2005) recently argued that the anisotropic distribution of the MW dwarf satellites presents a serious challenge to the standard CDM structure

1 Kavli Institute for Cosmological Physics and Department of Astronomy and Astrophysics, The University of Chicago, 933 East $56^{\text {th }}$ Street, Chicago, IL 60637 USA; zentner@kicp. uchicago.edu, andrey@oddjob.uchicago.edu

2 The Enrico Fermi Institute, 5640 South Ellis Ave., The University of Chicago, Chicago, IL 60637 USA

3 Department of Astronomy, The Ohio State University, 140 W. $18^{\text {th }}$ Ave., Columbus, OH 43210 USA; ognedin@astronomy.ohio-state.edu

4 Astronomy Department, New Mexico State University, MSC 4500, P.O. Box 30001, Las Cruces, NM 880003-8001; aklypin@ nmsu. edu formation paradigm. Kroupa et al. (2005) reached this conclusion by assuming that the luminous satellites correspond to a randomly-selected subset of dark matter subhalos and by taking an isotropic subhalo distribution as their null hypothesis.

There have been a number of studies of anisotropy in satellite galaxy distributions outside of the Local Group. Holmberg (1969) found that satellites of spiral galaxies with projected separations $r_{\mathrm{p}} \lesssim 50 \mathrm{kpc}$ are preferentially located near the short axes of the projected light distributions of their host galaxies (the Holmberg Effect). However, several subsequent studies found little evidence for such a preferential alignment (e.g., Hawley \& Peebles 1975; Sharp et al. 1979; MacGillivray et al. 1982). Zaritsky et al. (1997) found a statistically significant anisotropy similar to that advocated by Holmberg (1969), but only at larger projected distances, $200 \mathrm{kpc} \lesssim r_{\mathrm{p}} \lesssim 500 \mathrm{kpc}$. In a more recent study of a large sample of satellite galaxies in the Two Degree Field Galaxy Redshift Survey (Colless et al. 2001), Sales \& Lambas (2004) also found evidence for preferential alignment of satellites along the minor axis of the central galaxy. However, Brainerd (2004) performed a similar study of satellites in the Sloan Digital Sky Survey (York et al. 2000; Strauss et al. 2002), finding satellites to be preferentially aligned near the long axes of host galaxies and the degree of anisotropy to be a rapidly decreasing function of separation from the host galaxy. Note that the anisotropy measured by Brainerd (2004) is opposite in sense to the anisotropy reported by Holmberg (1969), Zaritsky et al. (1997), and Sales \& Lambas (2004).

Properties of dwarf satellite dark matter (DM) halos (or subhalos) in MW-sized hosts have been the subject of several recent studies, which used a new generation of high-resolution dissipationless simulations not affected by the "overmerging" problem (e.g., Klypin et al. 1999a; Moore et al. 1999; Stoehr et al. 2002, 2003; De Lucia et al. 2004; Kravtsov et al. 2004; Gao et al. 2004b; Reed et al. 2004). One of the main results is that the radial distribution of subhalos is more extended than 
the distribution of DM (Ghigna et al. 1998; Colín et al. 1999; Ghigna et al. 2000; Springel et al. 2001; De Lucia et al. 2004; Gao et al. 2004a). The DM subhalos also appear to have a significantly more extended and shallower radial distribution compared to the observed distribution of satellite galaxies both in galactic halos (Taylor et al. 2003; Kravtsov et al. 2004) and in cluster halos (Diemand et al. 2004; Gao et al. 2004a; Nagai \& Kravtsov 2005). Despite significant progress in our understanding of halo substructure, the anisotropy of subhalo spatial distributions has, so far, not been studied in as much detail. Zaritsky et al. (1997) reported no detectable anisotropy in the projected satellite distribution in the simulations of Navarro, Frenk, $\&$ White $(1994,1995)$. However, this conclusion refers to the statistical, projected distribution of the most massive satellites obtained after stacking many galaxy-sized halos, rather than anisotropy of the satellite distribution within a single host halo. More recently, Knebe et al. (2004) studied anisotropy of the subhalo distribution in dissipationless simulations of clustersized hosts. These authors found that subhalo distribution is anisotropic and is aligned with the major axis of the matter distribution of the host.

In this paper, we study the anisotropy of satellite distribution in Galaxy-sized halos using high-resolution cosmological $\mathrm{N}$-body simulations of structure formation in the concordance flat $\Lambda \mathrm{CDM}$ cosmology. We show that an isotropic distribution is not the correct null hypothesis for testing the CDM paradigm. The mass distributions in CDM halos are generally triaxial rather than spherical. We demonstrate that subhalos of the size thought to host the MW dwarf satellites are distributed anisotropically about their host halos with subhalos preferentially located along the major axes of their hosts. We also show that the null hypothesis distribution taken by Kroupa et al. (2005) is incorrect even in the case of an isotropic underlying distribution. As we were completing this study, Kang et al. (2005) presented a similar study, considering in particular the question of whether the anisotropy of the MW satellite distribution is consistent with the hierarchical formation scenario. Although our approaches differ in detail, their conclusions are consistent with ours. We present a more extensive study of the satellite distribution, both in three dimensions and in two-dimensional projection. We also explore the physical mechanisms that create the anisotropy measured in the simulations and discuss the implications of our results on our understanding of galaxy formation.

This manuscript is organized as follows. In $\S 2$, we describe our numerical simulations and our analysis methods. In $\S 3$, we present results on the anisotropic distribution of subhalos in Galaxy-sized halos. We discuss our results and their implications in $\S 4$. Lastly, we summarize our main findings and draw conclusions in $\S 5$. In an Appendix, we discuss the prospects of studying satellite anisotropy in projection.

Throughout this paper, we refer to halos that are contained within the virial radii of still larger halos as subhalos or satellites and we refer to halos that are not contained within a larger halo as host halos.

\section{METHODS}

\subsection{Numerical Simulations}

We analyze a simulation of three Milky Way-sized DM halos formed in a standard, "concordance" $\Lambda \mathrm{CDM}$ cosmology with $\Omega_{M}=1-\Omega_{\Lambda}=0.3, h=0.7$, and $\sigma_{8}=0.9$. The simulation was performed with the Adaptive Refinement Tree (ART)
$N$-body code (Kravtsov et al. 1997; Kravtsov 1999). This simulation has been discussed previously by Klypin et al. (2001), and Kravtsov, Gnedin, \& Klypin (2004, hereafter KGK04). We briefly review some of the details in this section.

The simulation began with a uniform $256^{3}$ grid covering a comoving, cubic box of $25 h^{-1} \mathrm{Mpc}$ on a side. Higher force resolution was achieved in dense regions using an adaptive refinement algorithm. The grid cells were refined if the particle mass contained within them exceeded a certain, specified threshold value. The multiple mass resolution technique was used to set up the initial conditions. A Lagrangian region corresponding to two virial radii about each halo was re-sampled at the initial epoch of $z_{i}=50$ with the highest-resolution particles of mass $m_{\mathrm{p}}=1.2 \times 10^{6} h^{-1} \mathrm{M}_{\odot}$. The high mass resolution region was surrounded by layers of particles of increasing mass with a total of five particle species. Only the regions containing the highest-resolution particles were adaptively refined. The maximum level of refinement in the simulations corresponded to a peak formal spatial resolution of approximately $100 h^{-1} \mathrm{pc}$.

We define a halo radius $R_{180}$, by the sphere, centered on the particle with the highest density, within which the mean density is 180 times the mean density of the universe $\rho_{\mathrm{M}}$, so that the mass and radius are related by $M_{180}=4 \pi\left(180 \rho_{\mathrm{M}}\right) R_{180}^{3} / 3$. Our three host halos, which we refer to as halo $\mathrm{G}_{1}$, halo $\mathrm{G}_{2}$, and halo $\mathrm{G}_{3}$, have masses of $M_{180}=1.66 \times 10^{12} h^{-1} \mathrm{M}_{\odot}, 1.24 \times$ $10^{12} h^{-1} \mathbf{M}_{\odot}$, and $1.19 \times 10^{12} h^{-1} \mathbf{M}_{\odot}$, respectively, and these halos contain $\sim 10^{6}$ particles within their virial radii. ${ }^{5}$ Their virial radii are $R_{180}=298 h^{-1} \mathrm{kpc}, 278 h^{-1} \mathrm{kpc}$, and $281 h^{-1} \mathrm{kpc}$, respectively.

We identified halos and subhalos using a variant of the Bound Density Maxima algorithm (Klypin et al. 1999b). First, we compute the local density at each particle using a smoothing kernel of 24 particles and identify local maxima in the density field. Beginning with the highest density particles and stepping down in density, we mark each peak as a potential halo center and surround the peak by a sphere of radius $r_{\text {find }}=10 h^{-1} \mathrm{kpc}$. All particles within the sphere are excluded from further consideration as potential halo centers. The parameter $r_{\text {find }}$ is set according to the smallest objects that we aim to identify robustly. After identifying potential halo centers, we iteratively remove unbound particles. For host halos, the mass and radius are set according to a fixed overdensity as described above. For subhalos, the outer boundary is somewhat ambiguous and we adopt a truncation radius $r_{\mathrm{t}}$, at which the density profile becomes greater than a critical value of $\mathrm{d} \ln \rho / \mathrm{d} \ln r=-0.5$. This criterion is based on the fact that we do not expect density profiles of CDM halos to be shallower than this and, empirically, this definition is approximately equal to the radius at which the background density of host halo particles is equal to the density of particles bound to the subhalo.

Upon identifying halos, we assign each halo a mass and radius and use the halo particles to determine a circular velocity profile $V_{\text {circ }}(r)=\sqrt{G M(<r) / r}$, and the maximum circular velocity, $V_{\max }$. We choose to quantify the size of subhalos according to $V_{\max }$ because this quantity is measured more robustly and is not subject to the same ambiguity as a particular mass definition.

Halos $\mathrm{G}_{1}, \mathrm{G}_{2}$, and $\mathrm{G}_{3}$ have maximum circular velocities of $V_{\max } \simeq 213 \mathrm{~km} \mathrm{~s}^{-1}, V_{\max } \simeq 199 \mathrm{~km} \mathrm{~s}^{-1}$, and $V_{\max } \simeq 183 \mathrm{~km} \mathrm{~s}^{-1} \mathrm{re}-$ spectively. These halos were selected to reside in a well-defined

5 These halos were referred to as $B_{2}, C_{2}$, and $D_{2}$ respectively by Klypin et al. (2001). 
filament at $z=0$. The halos $\mathrm{G}_{2}$ and $\mathrm{G}_{3}$ are neighbors located at $425 h^{-1} \mathrm{kpc}$ (i.e., $\approx 610 \mathrm{kpc} \sim 2 R_{\mathrm{vir}}$ ) from each other. The configuration of this pair thus resembles that of the Local Group. The third halo is isolated and is located $\sim 2 \mathrm{Mpc}$ away from the pair. The three hosts have similar masses at the present but rather different mass accretion histories. Host $\mathrm{G}_{1}$ undergoes a spectacular multiple major merger at $z \approx 2$, which results in a dramatic mass increase on a dynamical time scale. Halos $\mathrm{G}_{2}$ and $\mathrm{G}_{3}$ increase their mass in a series of somewhat less spectacular major mergers which could be seen as mass jumps at $5<z<1$. All three systems accrete little mass and experience no major mergers at $z \lesssim 1$ (or lookback time of $\approx 8 \mathrm{Gyr}$ ) and thus could host a disk galaxy. Note, however, that halos $G_{1}$ and $\mathrm{G}_{3}$ experience minor mergers during this period.

\subsection{Modeling Luminous Satellites}

The relative scarcity of MW satellites compared to predicted subhalo counts suggests that a naïve comparison of subhalo populations to luminous galaxies may not be correct. This implies that in order to make a more meaningful comparison with the data, we should have a model to identify the DM subhalos in simulations that would host observable, luminous galaxies. We consider two alternatives.

The first dwarf galaxy formation model we consider was recently proposed by KGK04 (see their $\S 6$ for details). This semi-analytic model is based on the subhalo evolutionary tracks extracted from the simulations used in this study. The smallmass dwarfs are identified with the halos that either have accreted a large fraction of their mass prior to the epoch of reionization (see also Bullock, Kravtsov, \& Weinberg 2000; Ricotti $\&$ Gnedin 2004) or were relatively massive at high redshifts and could therefore retain most of their gas and form stars after reionization. Some objects in the latter category could lose most of their former mass due to tidal stripping and appear as relatively low-mass halos at the present epoch. The dwarf satellite galaxies in this model can thus be hosted by both massive and low-mass subhalos at $z=0$.

The physical ingredients of the KGK04 model include: (1) the suppression of gas accretion by the extragalactic UV background; (2) an observationally-motivated recipe for quiescent star formation; (3) a starburst mode of star formation after strong peaks in tidal forces (which are calculated selfconsistently from the simulations); and (4) an accounting for the inefficient dissipation of gas in halos with $T_{\text {vir }} \lesssim 10^{4} \mathrm{~K}$. The model can successfully reproduce the circular velocity function, the radial distribution, and the morphological segregation of the observed MW satellites, as well as the basic properties of galactic dwarfs such as their star formation histories, stellar masses, and densities. In our analysis, we use the same set of subhalos that were considered to be "luminous" according to this model in KGK04 and refer to them as luminous subhalos.

The second model assumes that the observed MW satellites are hosted by the most massive subhalos (Stoehr et al. 2002, 2003). In this model, it is conjectured that the masses of the subhalos in which the luminous dwarfs are embedded are significantly underestimated because the DM density profiles in the central regions of the subhalos have been affected by tidal interactions (Stoehr et al. 2002, 2003; Hayashi et al. 2003, see, however, Kazantzidis et al. 2004b). The maximum circular velocities of the Local Group dwarfs may thus be systematically underestimated in observations because they are derived from stellar velocity dispersion measurements within radii consider-

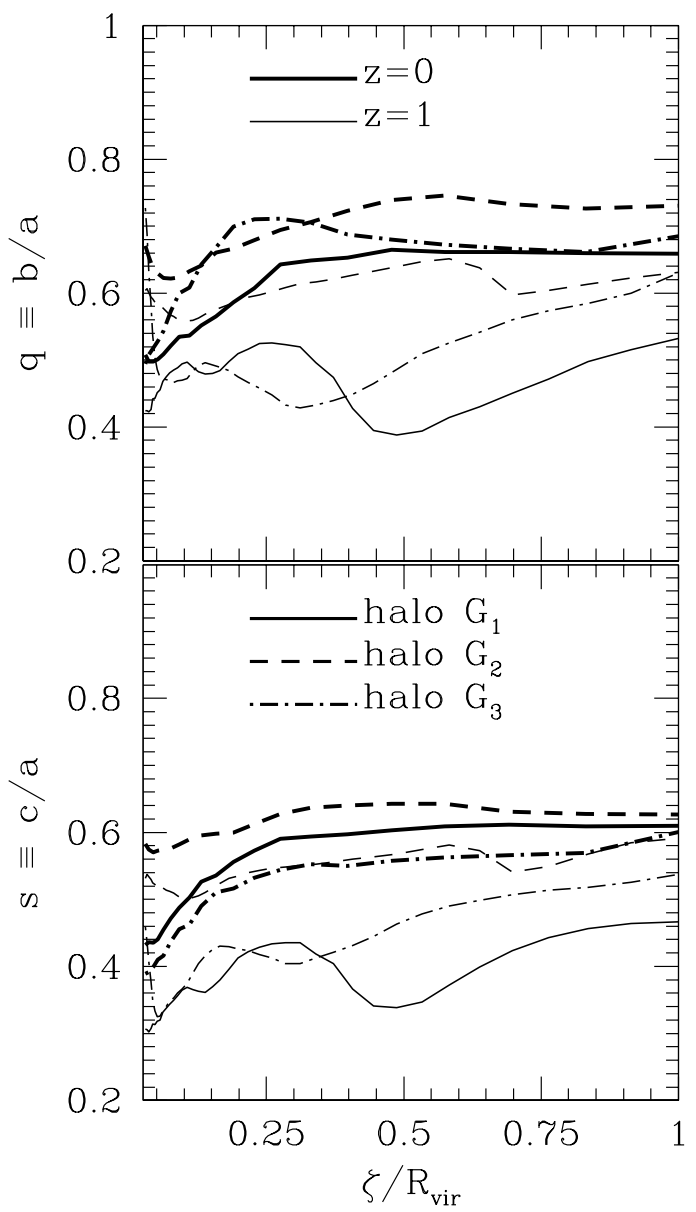

FIG. 1.- Host halo axis ratio profiles for the three MW-sized host halos. Top: intermediate-to-long axis ratio $q \equiv b / a$, as a function of long axis length. Bottom: short-to-long axis ratio $s \equiv c / a$. In both panels, the solid line represents halo $\mathrm{G}_{1}$, the dashed line represents halo $\mathrm{G}_{2}$, and the dot-dashed line represents halo $\mathrm{G}_{3}$. The thick lines represent the shape profiles at $z=0$, while the thin lines represent the halo shape profiles at $z=1$.

ably smaller than the radius at which $V_{\max }$ is achieved. Stoehr et al. (2002) argued that the bias induced by this is large, such that all of the observed MW satellites can be embedded in the most massive subhalos with $V_{\max } \geq 30-40 \mathrm{kms}^{-1}$. This model has an important physical implication. If the MW dwarfs inhabit the most massive subhalos, then there must exist a certain universal mass or $V_{\max }$ scale below which galaxy formation is completely quashed due to the UV background heating and inefficient gas cooling in dwarf halos. We consider this type of model by associating luminous dwarfs with the eleven subhalos with the highest values of $V_{\max }$ at $z=0$ within $300 \mathrm{kpc}$ of each MW-sized host halo.

\subsection{The Principal Axes of the Host Halos}

We determine the principal axes of the three simulated host halos and the corresponding principal axis ratios $q \equiv b / a$ and $s \equiv c / a(a>b>c)$ in the following way. We construct a modified inertia tensor given by (e.g., Dubinski \& Carlberg 1991)

$$
I_{i j}=\sum_{\nu} m_{\nu} x_{i}^{\nu} x_{j}^{\nu} / \zeta_{\nu}^{2}
$$

where $m_{\nu}$ is the mass of the $\nu$ th particle, $x_{i}^{\nu}$ is the $i$ coordinate with respect to a reference frame centered on the density peak of the halo, $\zeta_{\nu}^{2} \equiv\left(y_{1}^{\nu}\right)^{2}+\left(y_{2}^{\nu} / s\right)^{2}+\left(y_{3}^{\nu} / q\right)^{2}$, and $y_{i}^{\nu}$ are the particle coordinates with respect to the halo principal axes. We use 


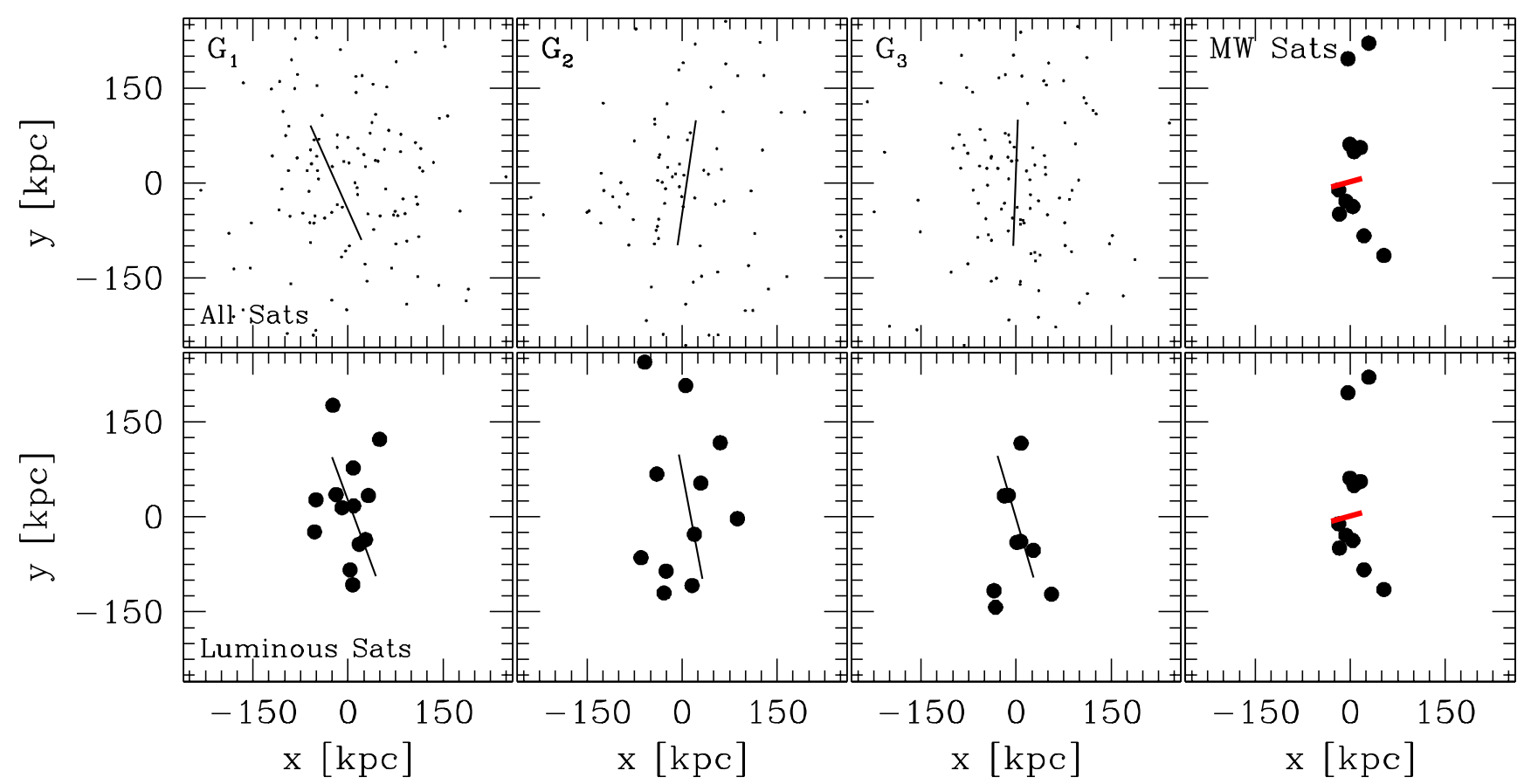

FIG. 2.- Projections of satellites in a plane orthogonal to their best-fit planes (see text). All panels show scatter plots of the positions of satellites projected onto a plane perpendicular to their best-fit plane. The best-fit plane corresponds to the vertical axis in this projection. In both rows, the first panel shows results for the subhalos of halo $G_{1}$, the second column for halo $G_{2}$, and the third column for halo $G_{3}$. The fourth column in each row shows the observed MW satellites. In the first three columns, the projection is such that the major axis of the host halo lies in the plane of the projection. In these panels, the major axis is shown as the thin, solid line. In the fourth column, the projection is such that the MW disk is seen edge-on and the MW disk orientation is denoted by the thick, solid line. In the top row, we compute the best-fit plane by considering all subhalos within $\approx 300 \mathrm{kpc}$ of the center of the host halo. In the bottom row, we compute the best-fit plane with respect to all luminous subhalos within $300 \mathrm{kpc}$ of the host halo center.

an iterative algorithm to determine the principal axes. We begin with the assumption of a spherical configuration $(a=b=c)$, construct the inertia tensor according to Equation (1), and diagonalize the tensor to determine the principal axes (eigenvectors) and the axis ratios (ratios of eigenvalues). We then repeat this process, using the results of the previous iteration to define the principal axes, until the results converge to a fractional difference of $10^{-3}$. The process generally takes fewer than 10 iterations to converge. The factor of $\zeta_{\nu}^{-2}$ in Eq. (1) serves to mitigate the influence of massive substructures at large distances, which can be a significant source of noise in the measurement (Dubinski \& Carlberg 1991).

In Figure 1, we show shape profiles at $z=0$ and $z=1$ for each host halo constructed in this way. The Figure shows the axis ratios as a function of the length of the major axis of the ellipsoid $\zeta$, and we construct profiles by considering all particles within this ellipsoid. In agreement with previous studies (e.g., Jing \& Suto 2002; Bullock 2002), all three MW-sized host halos are triaxial with $b / a \approx 0.6-0.7$ and tend to be more prolate than oblate with $c / b \approx 0.8-0.95$. Additionally, it is evident that halo shapes evolve as halos tend to be less spherical at high redshift, reflecting their younger dynamical age.

Currently, it is unclear whether such axis ratios are consistent with observational constraints on the shape of the MW halo. Recent studies suggest that the coherence of the Sagittarius tidal debris constrains the minor-to-major ratio to $c / a \gtrsim 0.8$ (Ibata et al. 2001; Majewski et al. 2003; Johnston et al. 2004). However, Helmi (2004a,b) argues that the Sagittarius tidal stream is consistent with a minor-to-major axis ratio as small as $c / a \simeq$ 0.6 (see also Martínez-Delgado et al. 2004) because the stream is dynamically young. Moreover, the shape constraint could be relaxed in a prolate halo where orbits along the long-axis are less susceptible to strong precession (see, however, Johnston et al. 2004). We note that the effect of baryon cooling during galaxy formation should make halos more spherical compared to halos in the dissipationless simulations analyzed here. The change in axis ratios is $\Delta(c / a) \sim 0.1-0.3$ in the inner regions of the halo, and it is not uncommon to see significant variation in axis ratios with $\zeta$ (Dubinski 1994; Kazantzidis et al. 2004a).

In our analysis below, we refer to subhalo positions in a coordinate system defined by the principal axes of the host halo inertia tensor, calculated using DM particles within the ellipsoid with $\zeta=0.3 R_{180}$ (for the MW-sized halos, this corresponds to $\zeta \approx 85 h^{-1} \mathrm{kpc} \approx 120 \mathrm{kpc}$ ). We choose particles with $\zeta<0.3 R_{180}$ in order to mitigate the influence of large substructures at large halo-centric distances on the reference frame definition; however, we find that the ellipsoids defined at different values of $\zeta$ are closely aligned at all radii within any single host, in agreement with Jing \& Suto (2002). We define the zenith angle, $0 \leq \theta \leq \pi / 2$, as the angle from the major axis of the halo. We also make analogous computations in two-dimensional projection, in which case we define a polar coordinate system with angle $\phi$, defined as the angle away from the major axis of the two-dimensional projected DM distribution.

\subsection{Satellite Planes}

Instead of defining satellite positions in the coordinate system set by the mass distribution of their host halo, which is difficult to determine observationally, one can construct coordinate systems with respect to the satellites themselves. Kroupa et al. (2005) found the MW satellites to be in a nearly planar distribution, so we follow the method of Kroupa et al. (2005) 


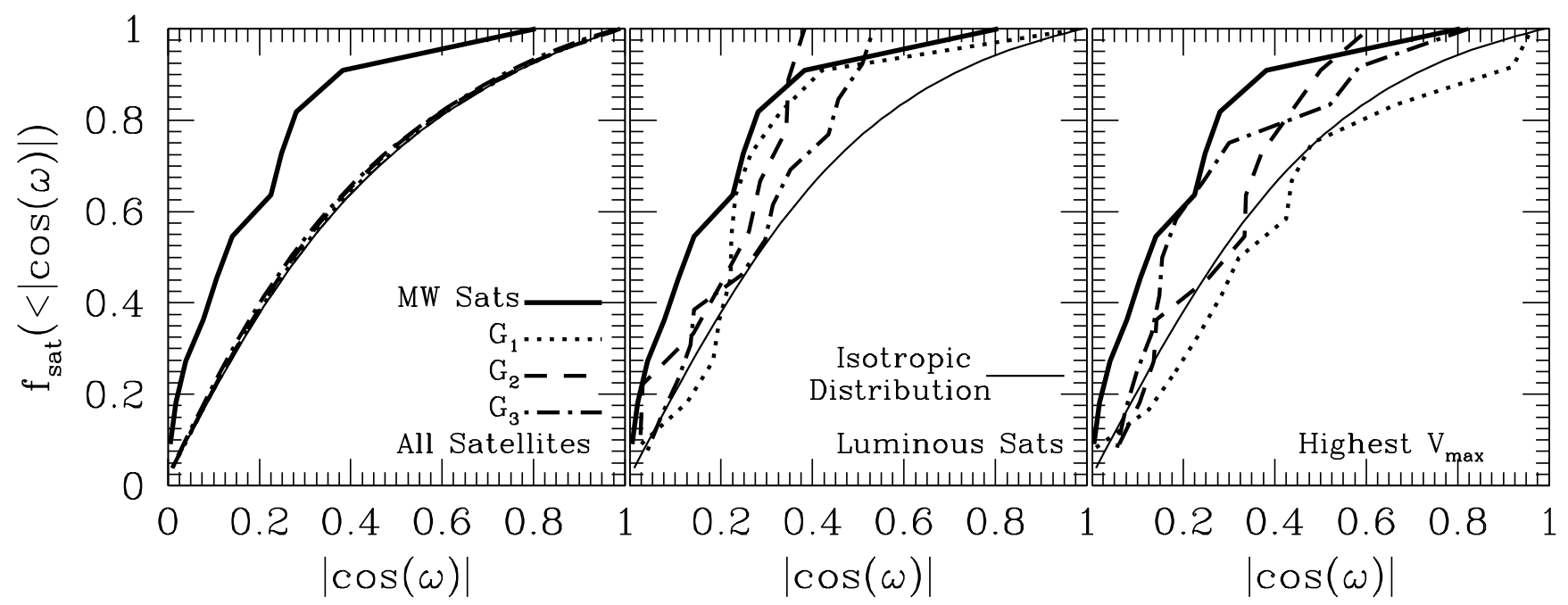

FIG. 3.- The cumulative fraction of halos with an angular position $<|\cos (\omega)|$ with respect to the normal to the best-fit plane as a function of $|\cos (\omega)|$. The left panel shows the distribution for all subhalos with $V_{\max } \geq 12 \mathrm{~km} \mathrm{~s}^{-1}$, the center panel shows the distribution of "luminous" satellites (see $\S 2.2$ ), and the right panel shows the distribution for the 11 subhalos with the largest $V_{\max }$ in each host. In each panel, the dotted lines represent halo $\mathrm{G}_{1}$, the dashed lines represent halo $\mathrm{G}_{2}$, and the dot-dashed lines represent halo $\mathrm{G}_{3}$. The thin, solid lines in each panel represent the expected CDF for an isotropic satellite distribution.

and find best-fit planes to the satellite positions in our host halos. Specifically, we determine a best-fit plane by minimizing the root-mean-square (rms) of the perpendicular distances of all satellites to the plane,

$$
D_{\mathrm{rms}}=\sqrt{\frac{\sum_{\mu=1}^{N}\left(\hat{n} \cdot \vec{x}_{\mu}-d\right)^{2}}{N}} .
$$

In Eq. (2), $\hat{n}$ is a unit vector normal to the plane, $d$ is the perpendicular distance from the origin (in this case, the origin is the Galaxy or the halo center) to the plane, $\vec{x}_{\mu}$ is the position of the $\mu$ th satellite, and the sum is over all $N$ satellites. We define a polar angle $0 \leq \omega \leq \pi / 2$, as the angle between a satellite position vector $\vec{x}_{\mu}$, and the best-fit unit vector $\hat{n}$, set at the point on the plane that minimizes the distance to the origin. Additionally, the value of $D_{\text {rms }}$ itself can also be used as a measure of the planarity of the satellite distribution (Kang et al. 2005), and we consider this statistic below.

\section{RESULTS}

\subsection{Visual Impression}

Figure 2 gives a visual impression of the planarity of the observed MW satellites, as well as the subhalos and the luminous subhalos in our simulations. Here and below, we consider all eleven known MW satellites within $R_{\max }=300 \mathrm{kpc}$ (Grebel et al. 2003). The MW satellites are shown in the rightmost panels of Fig. 2. The planarity of the distribution, noted by Kroupa et al. (2005) is very clear. Note, however, that recent studies indicate that the poles of the orbits of Ursa Minor (Piatek et al. 2005), as well as Sculptor and Fornax (Piatek et al., in preparation), are not coincident with the pole of the plane of MW satellites, nor are they coincident with each other. This is an indication that the high degree of planarity of the MW dwarfs may be transitory.

In the top row of Fig. 2, we show projections of all subhalos of each host onto the plane perpendicular to their best-fit plane. In these projections the best-fit plane runs vertically along the axis $x=0$ and we show the projected direction of the major axes in each panel by a thin solid line. In the bottom row, we show similar projections for the luminous subhalos. The spatial distributions of the highest- $V_{\max }$ subhalos are similar to those of the luminous subhalos.

The most obvious feature in all of the panels is that the bestfit plane of subhalos is strongly correlated with the major axis of the DM distribution of the host halo. If we draw $10^{5}$ random permutations of eleven subhalos from the full sample, the probability that the best-fit plane of any such subsample is inclined by more than $45^{\circ}$ from the host halo major axis is only $P \simeq 9 \%$, signaling anisotropy of the subhalo population as we discuss in more detail below. Correspondingly, the best-fit planes for the luminous subhalos are also aligned with the major axes of their hosts. Their degree of planarity is visually comparable to that of the MW satellites. In the following subsections, we quantify the anisotropy of satellite distribution.

\subsection{Satellite Planarity}

Kroupa et al. (2005) argued that the planarity of the observed MW satellites is inconsistent with the CDM paradigm based on their distribution in $|\cos (\omega)|$. However, their conclusion is incorrect for two reasons. First, as we show below, CDM does not predict that dwarf-sized subhalos are distributed isotropically. Second, the null hypothesis for the isotropic distribution for $|\cos (\omega)|$ used by Kroupa et al. (2005) is correct only in the limit of large sample size. This is because for a small number of objects, the distribution of $|\cos (\omega)|$ is not related to a fixed reference frame, but a frame determined by the selected objects themselves. This modifies the underlying cumulative distribution function (CDF) for $|\cos (\omega)|$. Kroupa et al. (2005) computed the distribution of $\omega$ for a single sample of $10^{5}$ subhalos, the result of which is a nearly uniform distribution in $|\cos (\omega)|$ on the interval $[0,1]$. However, the $|\cos (\omega)| \mathrm{CDF}$ for small number of objects drawn from the isotropic distribution is different. For example, consider the limit of randomly selecting precisely three objects from any underlying distribution. The three objects will always lie on the best-fit plane, such that $|\cos (\omega)|=0$ for all objects, independent of their underlying distribution.

In order to account for the small sample size, we generate the 


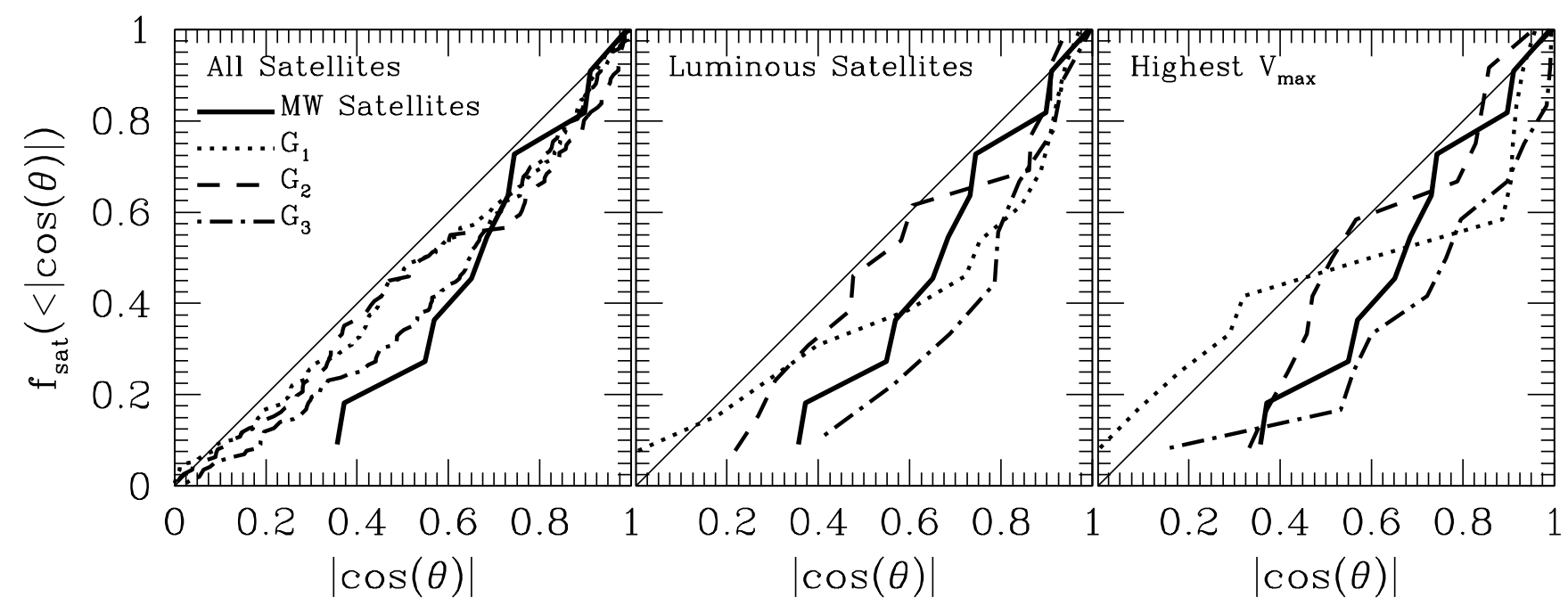

FIG. 4.- The cumulative fraction of satellites with the absolute value of the cosine of the zenith angle $<|\cos (\theta)|$. The zenith angle, $0 \leq \theta \leq \pi$, is defined as the angle from the major axis of the DM distribution of the host. The left panel shows the distribution for all subhalos with $V_{\max } \geq 12 \mathrm{~km} \mathrm{~s}^{-1}$. The center panel shows the distribution of only those subhalos that are "luminous" according to the model of Kravtsov et al. (2004, see $\S 2.2)$. The right panel shows the distribution for the eleven subhalos with the largest $V_{\max }$ in each host. The observed MW satellites (thick solid line) are placed on this plot by defining $\theta$ to be the angle from the pole of the MW disk. In each panel, the dotted lines represent halo $\mathrm{G}_{1}$, the dashed lines represent halo $\mathrm{G}_{2}$, and the dot-dashed lines represent halo $\mathrm{G}_{3}$.

distribution in $|\cos (\omega)|$ that should be expected from drawing eleven satellites from a uniform distribution. First, we assume an underlying isotropic subhalo distribution with a radial number density profile $n(r) \propto 1 /\left(1+\left(r / r_{\mathrm{c}}\right)^{3}\right)$ with $r_{\mathrm{c}}=0.25 R_{180}$ (see Zentner et al. 2005, who found this to be a good description of subhalo distributions in simulations and their semi-analytic models). Our results are not sensitive to the particular assumptions that we make about the radial distribution. We then draw $10^{5}$ random samples of eleven satellites from this distribution, determine the best-fit plane for each sample, and compute $\cos (\omega)$ for each satellite and the corresponding CDF. The resulting average CDF of $|\cos (\omega)|$ is shown as the thin, solid line in Figure 3. Notice that the $|\cos (\omega)|$ distribution for eleven satellites selected from an isotropic distribution is significantly different from a uniform distribution in $|\cos (\omega)|$, which would be a diagonal line.

We can assess the probability that the observed MW satellites are drawn from an isotropic distribution using the CDF of $|\cos (\omega)|$ constructed as described above. The KolmogorovSmirnov (KS) probability to draw the observed distribution of MW satellites within $R<300 \mathrm{kpc}$ from an isotropic distribution is $P_{\mathrm{KS}}^{\mathrm{ISO}} \simeq 0.15{ }^{6}$

In a similar analysis, we draw $10^{5}$ random samples of eleven subhalos from the full, simulated subhalo populations $\left(V_{\max } \geq\right.$ $12 \mathrm{~km} \mathrm{~s}^{-1}$ and $R<300 \mathrm{kpc}$ ) and construct the CDFs for these samples as shown in Fig. 3. Comparing this distribution to the isotropic case gives a KS probability of $P_{\mathrm{KS}} \simeq 0.49$. However, as we noted above, the best-fit planes in the subhalo samples are inclined by less than $45^{\circ}$ with respect to the major axis of the host halo in $91 \%$ of the samples, while there is no such preferential alignment in the isotropic case. For the luminous subhalos, the KS probability that they are drawn from an isotropic distribution is $P_{\mathrm{KS}} \simeq 0.39$. Finally, the probability that the MW satellites are drawn from the distribution of all subhalos is $P_{\mathrm{KS}} \simeq 0.16$. Figure 2 and the analysis below show that the dis-

${ }^{6}$ If we were to follow Kroupa et al. (2005) and use the uniform CDF that results from a very large sample size, we find $P_{\mathrm{KS}}^{\mathrm{ISO}} \approx 2 \times 10^{-3}$, in good agreement with their analysis. tributions of both the MW satellites and subhalos are actually anisotropic. The conclusion is that for a small sample (eleven), the $|\cos (\omega)|$ distribution is non-discriminatory. Other statistics must be used to draw meaningful conclusions.

The problem of the reference frame uncertainty for small sample sizes can be avoided if the frame is fixed and does not depend on the objects. In particular, we can choose to define the angles with respect to the halo major axes, (i.e., the zenith angle $\theta$, defined in $\S 2.3$ ). We show the $\operatorname{CDF}$ of $|\cos (\theta)|$ for the simulated subhalos in the left panel of Figure 4 and the CDF for an isotropic distribution by the thin diagonal line. For all three of the simulated MW-sized hosts, the subhalos are clearly distributed anisotropically with a preferential alignment along the host halo major axis. The KS probability of drawing this subhalo population from an isotropic distribution is $P_{\mathrm{KS}}^{\mathrm{ISO}} \simeq 1.5 \times 10^{-4}$. This is in contrast to the result of Willman et al. (2004), who find an isotropic distribution of subhalos in a MW-sized halo simulated by Reed et al. (2003). We find the differential fraction of satellites per unit $x \equiv|\cos (\theta)|$ averaged over all three host halos to be sharply peaked and well described by

$$
\frac{\mathrm{d} f_{\text {sat }}}{\mathrm{d} x} \simeq 0.80+1.15 x^{4.75} .
$$

The thick, solid lines in each of the panels of Figure 4 show a similar distribution in zenith angle for the eleven observed MW satellites within $300 \mathrm{kpc}$. The orientation of the MW halo is unknown, so the MW satellites cannot be placed on this plot without making an assumption about the orientation of the halo with respect to the disk. In the reference frame in which the zenith angle $\theta$, is measured with respect to the Galactic pole (we discuss implications of this assumption in $\S 4$ ), the KS probability of drawing the MW satellites from a distribution that is isotropic about the Galactic center is $P_{\mathrm{KS}}^{\mathrm{ISO}} \simeq 0.07$, while the probability of drawing the MW satellites from the sample of CDM subhalos is $P_{\mathrm{KS}} \simeq 0.34$. Alternatively, if we assume that the MW satellites are aligned along the minor or intermediate axes, the probabilities for the subhalos and MW satellites to have the same parent distribution are $P_{\mathrm{KS}} \simeq 0.02$ and 
$P_{\mathrm{KS}} \simeq 0.03$, respectively.

For the subhalos that are luminous according to the KGK04 model, the preferential distribution at low zenith angles remains and the KS probability of for all luminous subhalos to be drawn from an isotropic underlying distribution is only $P_{\mathrm{KS}}^{\mathrm{ISO}} \simeq 0.02$. For the eleven highest- $V_{\max }$ subhalos in each host, the KS probability of being sampled from isotropic distribution is $P_{\mathrm{KS}}^{\mathrm{ISO}} \simeq 0.05$. Note that for such small samples of objects, the halo-to-halo variation in the zenith angle distribution is large.

A useful measure of planarity is the rms distance to the bestfit plane, $D_{\text {rms }}$ (Kang et al. 2005, see Eq. (2)). In agreement with Kroupa et al. (2005), we find that $D_{\mathrm{rms}}^{\mathrm{MW}} \simeq 26.4 \mathrm{kpc}$ for the eleven MW satellites within $300 \mathrm{kpc}$. For eleven satellites selected randomly from the isotropic distribution above, the mean value of the rms plane width in $10^{5}$ sample realizations is $D_{\mathrm{rms}}^{\mathrm{iso}} \simeq 72 \mathrm{kpc}$ and the probability of a distribution similar to the MW satellites is $P\left(D_{\mathrm{rms}}^{\text {iso }} \leq D_{\mathrm{rms}}^{\mathrm{MW}}\right) \simeq 5 \times 10^{-3}$. Similarly, for the subhalo realizations averaged over all three host halos, the mean width is $D_{\mathrm{rms}} \simeq 58 \mathrm{kpc}$ and $P\left(D_{\mathrm{rms}} \leq D_{\mathrm{rms}}^{\mathrm{MW}}\right) \simeq 0.02$. This shows that both MW satellites and DM subhalos are distributed anisotropically, but the observed distribution is somewhat more planar.

The radial distribution of observed satellites is, however, more centrally concentrated than that of the subhalos. The median distance of the MW satellites from the Galactic center is a factor of two smaller than the median distance of subhalos from the centers of their hosts (Taylor et al. 2003; Kravtsov et al. 2004). The luminous subhalos have a radial distribution that is similar to the MW satellites and represent a biased subsample of the overall subhalo population. The rms distance to the best-fit plane is sensitive to the radial extent of the population and we expect more centrally concentrated populations of objects to have smaller values of $D_{\text {rms }}$.

Indeed, we find that the peak of the distribution of $D_{\text {rms }}$ scales linearly with the median of the radial positions of the underlying population $R_{\text {med }}$. The rescaled rms distance to the plane, $\delta_{\mathrm{rms}} \equiv D_{\mathrm{rms}} / R_{\text {med }}$, can serve as a useful measure of planarity that normalizes out the radial extents of the different populations. In Figure 5, we show the probability distribution of selecting eleven satellites with a particular value of $\delta_{\text {rms }}$ drawn from the isotropic distribution and from the samples of subhalos of hosts $G_{1}, G_{2}$, and $G_{3}$. For both the isotropic distribution and for the simulated subhalos, $R_{\text {med }} \approx 167 \mathrm{kpc}$. Figure 5 shows that in terms of the probability distribution of $\delta_{\mathrm{rms}}$, the observed value is not unusual. In fact, we find that the value of $D_{\mathrm{rms}}^{\mathrm{MW}}$ is not unlikely $\left(P\left(\delta_{\mathrm{rms}}^{\mathrm{iso}} \leq \delta_{\mathrm{rms}}^{\mathrm{MW}}\right) \simeq 0.14\right)$ even for a random sample of eleven objects drawn from an isotropic distribution, if their radii are rescaled to have the same $R_{\text {med }}$ as the MW satellites. The figure also shows that the distribution of subhalos is anisotropic, which shifts the probability distribution of $\delta_{\text {rms }}$, increasing the probability of selecting a subsample with a degree of planarity comparable to that of the MW satellites: $P\left(\delta_{\mathrm{rms}}^{\mathrm{sub}} \leq \delta_{\mathrm{rms}}^{\mathrm{MW}}\right) \simeq 0.30$.

In the model of KGK04, the luminous subhalos of $\mathrm{G}_{1}$ and $\mathrm{G}_{3}$ match the observed radial distribution of the MW satellites well. We thus expect that their $D_{\text {rms }}$ will be comparable to the observed value. Indeed, we find for the luminous subhalos that $D_{\mathrm{rms}} \simeq 29.4 \mathrm{kpc}, D_{\mathrm{rms}} \simeq 46.2 \mathrm{kpc}$, and $D_{\mathrm{rms}} \simeq 27.4 \mathrm{kpc}$ for hosts $\mathrm{G}_{1}, \mathrm{G}_{2}$, and $\mathrm{G}_{3}$, respectively. These values are similar to $D_{\mathrm{rms}}^{\mathrm{MW}}$. The values of $\delta_{\text {rms }}$ for the MW satellites and the luminous subhalos are shown by arrows in Figure 5.

These results indicate that the main reason that the $M W$ satel-

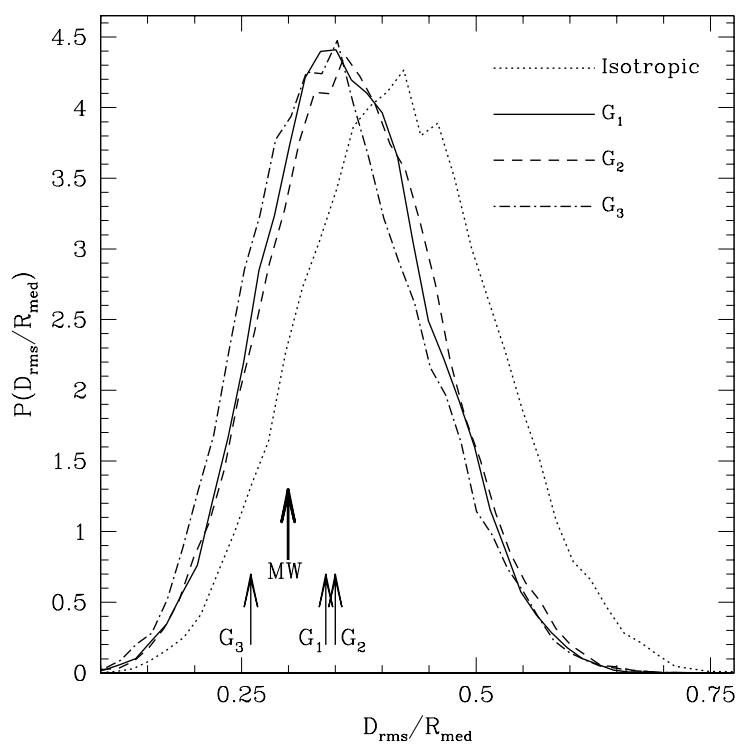

FIG. 5.- The distribution of the rms dispersions of satellite distribution around their best fit planes. The dispersions are rescaled to the median distance of each population to the center of their host (see text for details). The dotted line corresponds samples of 11 objects selected from an isotropic population distributed radially as the simulated subhalos. The other lines corresponds to samples of 11 subhalos drawn from the subhalos of $\mathrm{G}_{1}$ (solid line), $\mathrm{G}_{2}$ (dashed line), and the $\mathrm{G}_{3}$ (dot-dashed line). The labeled arrows at the bottom of the plot mark the values of $D_{\mathrm{rms}} / R_{\mathrm{med}}$, for the observed MW satellites and the luminous subhalos of $\mathrm{G}_{1}, \mathrm{G}_{2}$, and $\mathrm{G}_{3}$.

lites occupy a narrower plane than DM subhalos is their more centrally-concentrated radial distribution. Given that there are physical reasons to expect such radial bias (KGK04), we conclude that the observed anisotropy of the MW satellites is consistent with CDM predictions. However, we note that this agreement requires approximate alignment of the major axis of the halo that hosts the MW and the pole of the MW disk. In the next section, we discuss the origin of the subhalo anisotropy and the implications of such disk-halo alignment.

\section{DISCUSSION}

\subsection{Origin of the Subhalo Anisotropy}

It is interesting to ask why satellite halos should have a strongly-anisotropic distribution aligned with the major axis of the host halo.

Several suggestions have been made to explain the anisotropy observed by Holmberg (1969) and Zaritsky et al. (1997). Quinn \& Goodman (1986) investigated the effect of enhanced dynamical friction for orbits that are nearly co-planar with a galactic disk. The idea is that nearly co-planar orbits would be driven toward the disk plane (e.g., Binney 1977) and decay more rapidly due to additional interactions with the disk component. In this way, satellites on nearly co-planar orbits would be preferentially cannibalized by the disk and these orbits depopulated. For example, such cannibalized dwarf galaxies can significantly contribute to the formation of the thick disk (Abadi et al. 2003). However, the conclusion of Quinn \& Goodman (1986) was that this process is not efficient enough to account for the results of Holmberg (1969) for satellites closer than $\sim 50 \mathrm{kpc}$. Peñarrubia et al. (2002) extended this argument to include the effect of an oblate DM halo. They found this process to be efficient only within $\sim 50 \mathrm{kpc}$ of the host due to 
HALO $G_{1}$

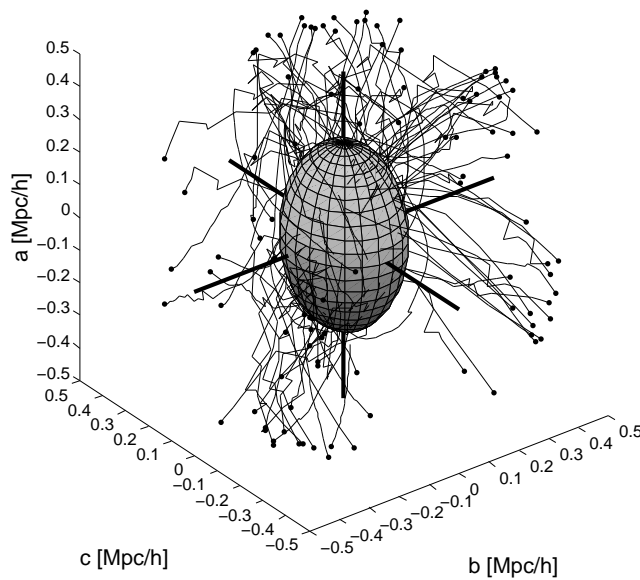

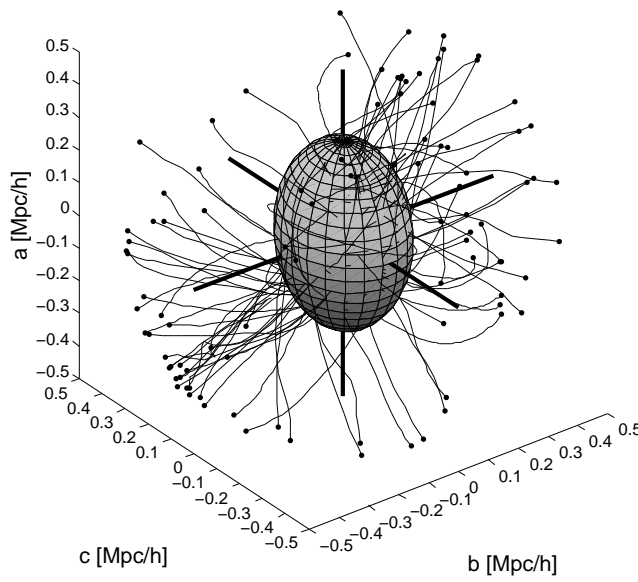

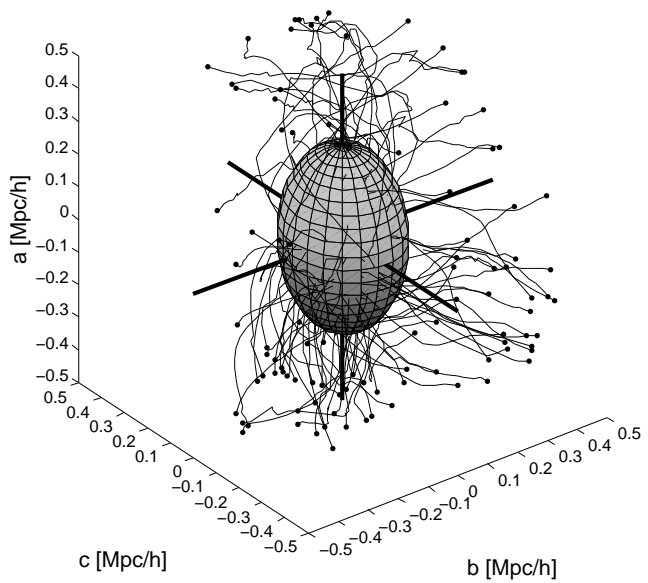

FIG. 6. - Trajectories of 100 randomly selected subhalos accreting onto host halos $\mathrm{G}_{1}, \mathrm{G}_{2}$, and $\mathrm{G}_{3}$ (panels from left to right). The trajectories are plotted relative to a coordinate system centered on the most bound particle in the parent halo and oriented along the principal axes of the parent halo evaluated at $z=0$. The coordinates

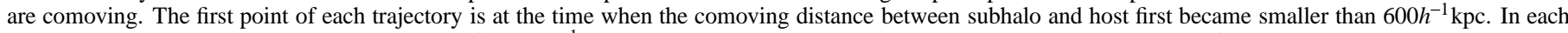

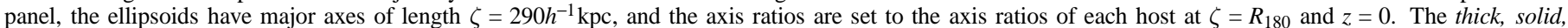

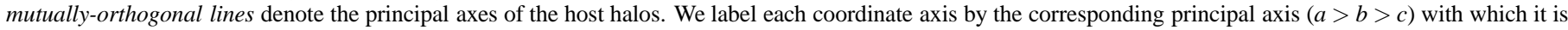
aligned. Preferential accretion along the direction of the long axis is evident for halos $\mathrm{G}_{1}$ and $\mathrm{G}_{3}$.

considerably longer orbital decay times at larger radii (Zaritsky \& White 1994; Zaritsky \& Gonzalez 1999). Note that the MW-sized halos in our simulations are prolate, so this process should be even less efficient. Yet, the anisotropy of satellites is present.

Two possibilities are that the anisotropy reflects a direction of preferential infall set by the environment (e.g., Tormen 1997) and/or that there is some other dynamical process that drives evolution toward anisotropy after accretion onto the host halo. Knebe et al. (2004) investigated the first possibility for cluster halos to address the anisotropy observed in systems such as the Virgo cluster (e.g. West \& Blakeslee 2000). Knebe et al. (2004) concluded that preferential accretion along the directions of filaments accounts for much of the bias in satellite orbits in cluster-sized systems. This result does not extend trivially to MW-sized systems. Clusters are rare, highly-biased objects that generally form relatively recently at the "nodes" of filaments that are comparable in thickness to the size of clusters themselves (e.g., Klypin \& Shandarin 1983; Bond et al. 1996; Colberg et al. 2004). It is therefore not surprising that many merging halos get "funneled" into clusters along dominant filamentary directions. Unlike clusters, MW-sized objects typically form earlier, are significantly less biased at the present epoch, and do not generally reside in filaments of comparable dimension. In this case, it seems that infalling substructure may be less likely to accrete along a single, dominant filamentary direction over the formation history of the halo. In addition, clusters have generally assembled their masses and accreted their satellites much more recently than galaxy-sized systems. As a result, many cluster subhalos are dynamically young and have undergone only a small number of orbits $(\lesssim 1-2)$ in the potential of the main halo, while the subhalos in our MW size halos have typically undergone several orbits within the main halo (see, e.g., KGK04).

In Figure 6, we show trajectories for 100 randomly-selected subhalos of each host halo as they are accreted. The trajectories are constructed from the 96 saved simulation timesteps by examining the $25 \%$ most bound particles in each halo. Halos at adjacent timesteps that share the highest common fraction of these particles are identified with each other, as described in $\S 4$ of KGK04. In two of the three cases (halos $G_{1}$ and $G_{3}$ ), there is clearly preferred satellite accretion along the direction of the major axis of the host halo. In the case of halo $\mathrm{G}_{2}$, the accretion is less anisotropic, but there appears to be a small preference for accretion toward the octant $a<0, b<0, c>0$. We have examined the accretion histories of the host halos more closely and halo $G_{2}$ appears to feed off of a filament that runs roughly in this direction during its early evolution $(z \gtrsim 1)$. It subsequently accretes along a filament that is more closely aligned with its major axis direction at $z=0$. Note that the halos $G_{1}$ and $G_{3}$ have both the highest degree of satellite anisotropy (see $\S 3$ ) and the most pronounced preferred accretion directions.

These results indicate that a preferential direction of satellite accretion is an important factor in determining the distribution of satellites in MW-sized halos, just as it is for cluster-sized hosts. Although filaments at the present time may be thick, they were significantly thinner in the past, when many of the surviving subhalos were accreted. Moreover, the matter distribution in filaments is concentrated toward the axis of the filament (e.g., Colberg et al. 2004). Finally, a fraction of subhalos may be accreted as members of groups, which are biased spatially and often located near the centers of filaments. That the preferential accretion direction is correlated with the major axis of the host halo is not surprising, because the major axis is typically determined by the direction of the most recent major merger.

In our DM-only simulations, effects like the cannibalization of satellites on co-planar orbits by material associated with the disk are absent, yet the substructure anisotropy is present, even for subhalos that have orbited within the host potential for many dynamical times. This is due to the fact that elongated potentials, similar to the potentials induced by our triaxial host halos, support orbits that make long excursions along the major axis of the potential (see Statler 1987). To illustrate this, we perform a simple, idealized experiment. We integrate the orbits of 200 


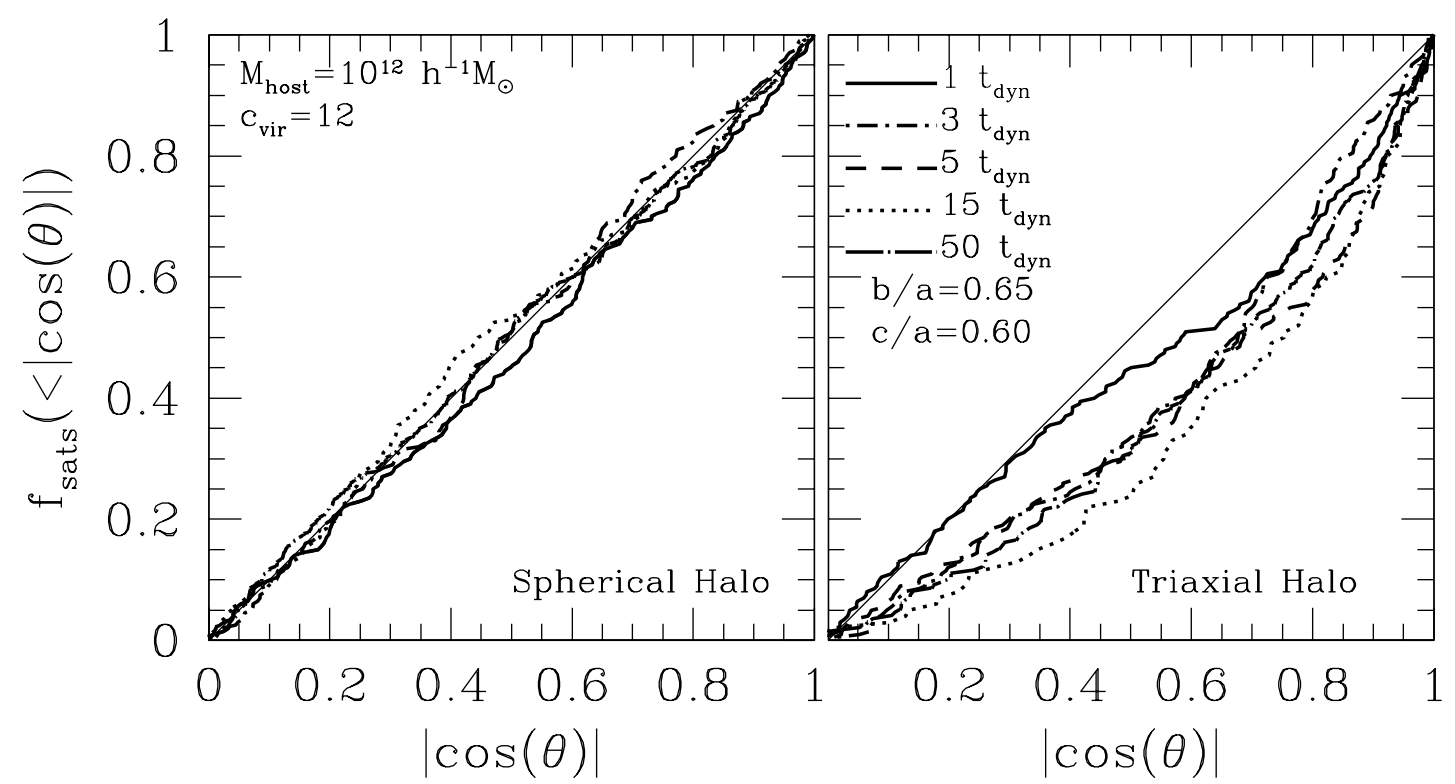

FIG. 7.- Generation of an anisotropic distribution of satellites in triaxial potentials. The distribution of zenith angle cosines for 200 isotropically accreted test particles at different epochs (lines of various type; the time is in units of the dynamical time of the host at the virial radius). The left panel represents control case of the spherical halo potential, while in the right panel the host has ellipsoidal isodensity contours with axis ratios of $b / a=0.65$ and $c / a=0.60$, similar to those of halos $\mathrm{G}_{1}, \mathrm{G}_{2}$, and $\mathrm{G}_{3}$. The fiducial host halo has a virial mass of $M_{180}=10^{12} h^{-1} \mathrm{M}_{\odot}$ and the NFW mass distribution with concentration parameter of $c_{180}=12$. The solid line indicates an integration for $1 t_{\mathrm{dyn}}$, the dot-short-dashed line indicates integration for $3 t_{\mathrm{dyn}}$, the dashed line for $5 t_{\mathrm{dyn}}$, the dotted line for $15 t_{\mathrm{dyn}}$, and the dot-long-dashed line for $50 t_{\mathrm{dyn}}$. The thin solid line corresponds to the isotropic distribution. The figure shows that in the spherical potential tracer distribution remains isotropic at all times. In this case of prolate, triaxial potential, the orbits evolve toward an anisotropic distribution similar to that of the observed satellites and simulated subhalos.

test particles in the static potential of a triaxial generalization of the density profile of Navarro et al. (1997, NFW hereafter),

$$
\rho(\zeta) \propto\left(\frac{\zeta}{r_{\mathrm{s}}}\right)^{-1}\left(1+\frac{\zeta}{r_{\mathrm{s}}}\right)^{-2}
$$

with ellipsoidal contours of constant density. $\zeta$ is defined as in $\S 2.3$. For simplicity, we choose constant axis ratios $b / a=0.65$ and $c / a=0.6$, similar to our simulated host halos (Fig. 1), and a halo concentration $c_{180}=R_{180} / r_{\mathrm{s}}=12$, which is typical of MWsized halos (Bullock et al. 2001). We choose the initial velocities for each orbit according to the distribution of initial conditions for subhalos presented in Zentner et al. (2005). In order to demonstrate the influence of the triaxial potential, we assumed spherically symmetric infall, rather than the anisotropic infall depicted in Figure 6.

The results of this exercise are shown in Figure 7, where we plot the distribution of subhalo zenith angle for the subhalos in the triaxial model and a spherical model that serves as a control. We integrate the orbits over an interval of $50 t_{\mathrm{dyn}}$, where $t_{\text {dyn }}$ is the dynamical time of the model at $R_{180}$. The net effect is clear and not surprising, but is often neglected. The prevalence of orbits that make long excursions along the major axis of the potential induces and maintains an anisotropic distribution of test particles. The test particles assume a distribution consistent with the triaxiality of the potential and, in fact, in a live, triaxial density distribution, it is the presence of these orbits that maintain the triaxial shape of the system (Gerhard \& Binney 1985; Udry \& Martinet 1994; Barnes \& Hernquist 1996; Merritt \& Quinlan 1998; Valluri \& Merritt 1998).

We neglected many effects in this experiment. These include the influence of the subhalos on the host potential, and the growth and evolution of the host halo potential that occurs in cosmological simulations. As we show in Figure 1, the host halo shapes evolve from $c / a \sim 0.4$ at $z \sim 1$ to $c / a \sim 0.6$ at $z \sim 0$. Moreover, subhalos on elongated orbits will be more vulnerable to tidal disruption because they generally pass closer to the center of the potential where the tides are strongest (e.g., Zentner $\&$ Bullock 2003; Zentner et al. 2005). The net effect should be that such orbits will be gradually depopulated. Nevertheless, our experiment illustrates that regardless of anisotropic infall, the satellite distribution can develop anisotropy due to the triaxiality of the host potential, even in the absence of any enhanced destruction due to the central disk.

\subsection{Anisotropy of the globular cluster distribution}

As we pointed out above, our results indicate that the observed distribution of the MW dwarf satellite galaxies is generally consistent with CDM predictions if the major axis of the MW DM halo is approximately aligned with the Galactic pole. If this is the case, based on our discussion of orbital structure in the previous section, we should expect similar anisotropies to exist in other populations that may serve as test particles in the MW halo potential.

Consider the distribution of the MW globular clusters (GCs). Frenk \& White (1982) studied a subsample of the MW GCs and concluded that both metal-rich and metal-poor globular cluster systems are slightly flattened. Subsequently, Zinn (1985) used a larger GC sample and concluded that metal-rich GCs are in a disk-like configuration (see also Armandroff 1989), while the distribution of the metal-poor clusters is nearly spherical. More recently, Hartwick (2000) analyzed the distribution of 15 metalpoor, distant globular clusters $(R \gtrsim 25 \mathrm{kpc}$ and $[\mathrm{Fe} / \mathrm{H}]<-1)$ and found that they form a flattened system with a minor axis highly-inclined relative to the MW disk rotation axis.

Following Zinn (1985), we divide the GC sample of Harris 


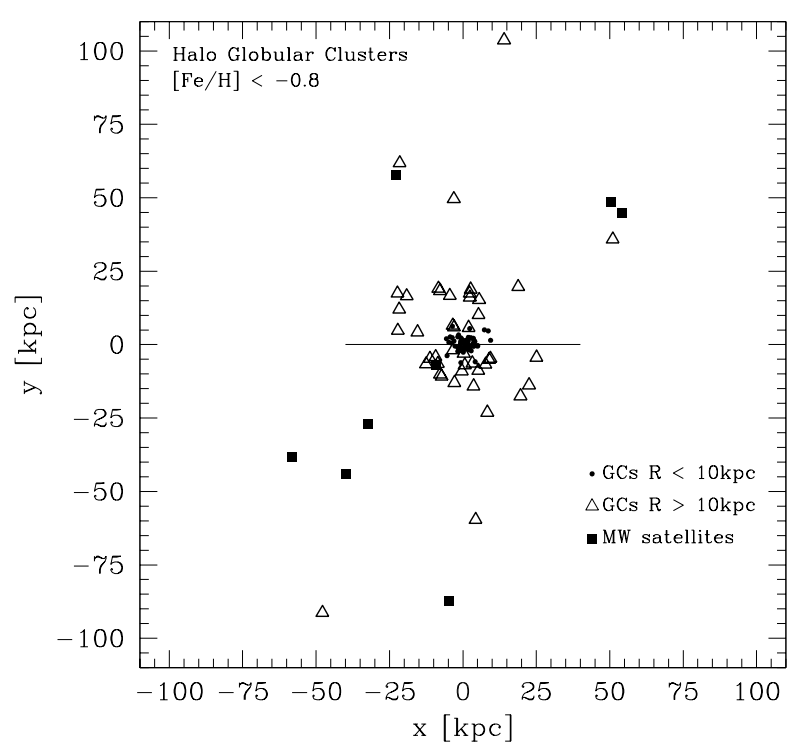

FIG. 8. - The distribution of metal-poor, MW globular clusters $([\mathrm{Fe} / \mathrm{H}]<$ $-0.8)$ and dwarf satellite galaxies. All positions are projected onto a plane perpendicular to the best-fit plane of the 44 most distant globulars $(R>10 \mathrm{kpc})$. The points correspond to the GCs with $R<10 \mathrm{kpc}$. The open triangles correspond to GCs with $R>10 \mathrm{kpc}$. For comparison, we show the positions of the MW satellite galaxies as the filled squares. The thin, solid, horizontal line represents the orientation of the MW disk. Note that only 8 of the MW satellites fall within the limits of this plot and the projection is oriented at an angle of nearly $36^{\circ}$ with respect to their best-fit plane.

$(1996)^{7}$ into halo and disk clusters using a metallicity threshold. We consider the distribution of metal-poor $([\mathrm{Fe} / \mathrm{H}] \leq-0.8)$, halo GCs. The distribution of the metal-poor GCs is shown in Figure 8 along with the distribution of the innermost MW dwarf satellites. Visually, the distribution of these two populations of halo objects appear to have similar anisotropy.

Just as for the dwarf satellites, we compare the distribution of GCs to an isotropic distribution using the distribution of $|\cos (\theta)|$, where $\theta$ is defined relative to the Galactic pole, and $\delta_{\text {rms }}^{\mathrm{GC}}$ because $|\cos (\omega)|$ has little discriminatory power. The results are summarized in Table 4.2. The inner globular clusters $(R<10 \mathrm{kpc})$ appear to lie in a flattened distribution $\left(P\left(\delta_{\mathrm{rms}}^{\mathrm{iso}}<\right.\right.$ $\left.\delta_{\mathrm{rms}}^{G C}\right) \simeq 0.02$ ) that is closely aligned with the disk plane. The normal to the best-fit plane of inner halo GCs is offset form the MW pole by only $\Theta_{\mathrm{MW}} \simeq 17^{\circ}$. Similar to Hartwick (2000), we find that the distant halo GCs show marginal evidence for a distribution that is aligned with the pole of the MW disk $\left(\Theta_{\mathrm{MW}} \simeq 80^{\circ}\right)$, a configuration that is consistent with the distribution of the MW dwarf satellites.

An additional piece of evidence comes from the distribution of the M31 satellites. In this case, distances to the M31 subgroup have considerably larger errors than the MW dwarfs, so these objects carry less statistical weight. Nevertheless, Hartwick (2000) studied the satellites of M31 in detail and found that, like the MW satellites, they appear to be arranged in a prolate structure that has its best-fit major axis misaligned with the pole of the M31 disk by only $\sim 20^{\circ}$. The distributions of M31 and MW satellites appear to show a similar anisotropy with preferential alignment along the galactic poles.

\subsection{Implications}

7 The globular cluster catalog is available at URL http://physwww. physics.mcmaster.ca/ $\sim$ harris/mwgc. dat.
ANISOTROPY OF THE METAL-POOR MILKY WAY Globular Cluster Distribution

\begin{tabular}{ccccc}
\hline \hline$R[\mathrm{kpc}]$ & $\Theta_{\mathrm{MW}}$ & $\Theta_{\mathrm{SATS}}$ & $P_{\mathrm{KS}}^{\theta}$ & $N_{\mathrm{GC}}$ \\
\hline $0-10$ & $17^{\circ}$ & $79^{\circ}$ & 0.12 & 68 \\
$>10$ & $80^{\circ}$ & $36^{\circ}$ & 0.03 & 44 \\
\hline
\end{tabular}

Column description: (1) the range of galacto-centric distances in GC subsample; (2) $\Theta_{\mathrm{MW}}$, the angle between the MW pole and the unit vector normal to the best-fit plane of GCs in the sample $\left(\Theta_{\mathrm{MW}}=0 \mathrm{deg}\right.$ corresponds to the Galactic disk plane, while $\Theta_{\mathrm{MW}}=90$ deg corresponds to a plane parallel to the MW pole); (3) $\Theta_{\text {SATS }}$, the angle between the best-fit plane of GCs and the best-fit plane of MW dwarf satellite galaxies; (4) $P_{\mathrm{KS}}^{\theta}$, the KS probability for the GC sample to be drawn from an isotropic distribution using the cumulative distribution of $|\cos (\theta)|$, where $\theta$ is the angle with between position vector of the satellite and Galactic pole; (5) the number of halo GCs in each subsample.

The angular distribution of satellites and its interpretation in the context of the anisotropy of the CDM subhalos have important implications for our understanding of galaxy formation. Collapsing halos acquire angular momentum via interactions with quadrupolar mass density fluctuations (Peebles 1969; Doroshkevich 1970; Efstathiou \& Jones 1979; Barnes \& Efstathiou 1987). In the simplest scenario of disk galaxy formation, baryons in halos begin by sharing the angular momentum distribution of the DM, on average, conserving it as they cool and condense (e.g., Fall \& Efstathiou 1980). This leads to a picture where the poles of disk galaxies are collinear with the net angular momentum vectors of their host halos, which are generally aligned with halo minor axes (e.g., Warren et al. 1992; Porciani et al. 2002; Faltenbacher et al. 2005).

van den Bosch et al. (2002) studied the alignment of the angular momenta of DM halos and their baryons in adiabatic simulations, and found that they are generally well aligned, with a distribution for the angular misalignment that is sharply peaked between $\sim 10^{\circ}-20^{\circ}$, but with an extended tail to larger angles such that the median is $\sim 30^{\circ}$. Chen et al. (2003) found similar results in adiabatic simulations and models with radiative cooling. Both van den Bosch et al. (2002) and Chen et al. (2003) measured increasing misalignment with decreasing halo mass and speculated that the extended tail could be partially due to noise in the measurement of angular momenta in small objects. We note that Kazantzidis et al. (2004a) found the gaseous disk of a young $(z \gtrsim 4)$ galaxy progenitor in their simulation to be aligned nearly perpendicular to the major axis of the DM halo, which is the alignment that our results suggest.

In this paper, we have shown that the predicted spatial distribution of CDM subhalos is consistent with the distribution of the MW satellites, if the pole of the MW disk is nearly aligned with the major axis of its outer DM halo. This requires a more complicated disk formation scenario where the halo and baryons mutually adjust as they evolve toward a stable configuration. It would be interesting to explore whether such an alignment is supported by observational evidence from other galaxies and numerical models of galaxy formation. Along these lines, we show the anisotropic, two-dimensional projected subhalo distributions in the Appendix. 
As we noted in the introduction, current observational results are contradictory. Holmberg (1969), Zaritsky et al. (1997), and Sales \& Lambas (2004) report that satellites of other galaxies exhibit statistical anisotropy similar to that of the MW dwarfs. Specifically, they find satellites to be preferentially located near the minor axes of the projected light distributions of host galaxies. However, the study of Brainerd (2004) shows evidence of the opposite correlation of satellite position with the major axis of their host galaxy.

One potential avenue for checking the disk-halo alignment is weak lensing. Hoekstra et al. (2004) recently presented the first weak lensing measurement of halo ellipticity obtained under the assumption that the halo mass and galaxy light distributions are aligned. This is likely a sound assumption for earlytype galaxies, which may indeed dominate the lensing signal in a sample of mixed morphological types. However, if galactic disks are preferentially aligned orthogonal to the major axes of their halos, the alignment for late-type systems would be opposite to that assumed by Hoekstra et al. (2004). Observational tests should be possible with the large dataset of the Sloan Digital Sky Survey.

On the theoretical side, Navarro, Abadi, \& Steinmetz (2004) recently argued that the angular momenta of galactic disks are aligned perpendicular to the minor axis and parallel to the intermediate axis of the inertia tensor of the surrounding matter distribution, both in their simulations of galaxy formation and in observations of nearby galaxies in the Local Supercluster. Unfortunately, it is not clear whether the major axes of galactic halos are always aligned with the intermediate axis of the surrounding large-scale structure. Navarro et al. (2004) also reported that the disks of nearby spirals tend to be oriented perpendicular to the Supergalactic plane. We generally expect the major axes of halos to be oriented along the filamentary structure of the Local Supercluster, so such a tendency is consistent with a scenario where disks orient themselves perpendicular to halo major axes.

The shape of the halo and the orbits of satellites within prolate and triaxial hosts may have important consequences for disk heating and the build up of the stellar halo. For example, given that the orbital energies of most surviving subhalos are relatively large, one may expect that the number of satellite passages close to the galactic center within the last $\sim 10 \mathrm{Gyr}$ should be small (e.g., Font et al. 2001). However, a satellite on a box-like orbit can pass arbitrarily close to the disk. This may enhance both the heating of the stellar disk (e.g., Tóth \& Ostriker 1992; Huang \& Carlberg 1997; Ibata \& Razoumov 1998; Moore et al. 1999; Velázquez \& White 1999) and the efficiency of tidal disruption of satellites and the formation of the stellar halo (e.g., Helmi \& White 1999; Bullock et al. 2001). Objects that experience close passages will be preferentially disrupted during halo evolution, such that it may appear that the presentday halo is devoid of satellites capable of doing damage. These considerations imply that the triaxiality of host potentials has to be taken into account in semi-analytic and numerical models of subhalo evolution (e.g., Bullock et al. 2000; Taylor \& Babul 2001, 2004; Benson et al. 2004; Zentner \& Bullock 2003; Zentner et al. 2005) in order for the treatment to be accurate.

Lastly, the alignment of satellite orbital planes along the halo major axis can have implications for the evolution of tidal tails. Helmi (2004a,b) argues that the data on the Sagittarius tidal debris (Majewski et al. 2004; Law et al. 2004) provide a strong indication that the MW halo is prolate with its major axis aligned with the Galactic pole and that the mean axis ratio within the orbit of Sagittarius is $0.65 \lesssim c / a \lesssim 0.8$. Again, this is the alignment necessary to explain the positions of the MW dwarfs. Note, however, that Johnston et al. (2004) argue that the precession of the orbital plane of Sagittarius implied by the data require an oblate halo that is, at most, slightly flattened $c / a \gtrsim 0.85$.

\subsection{Caveats}

One of the caveats to our results (and, indeed, to the results of the related studies of Knebe et al. (2004) and Kang et al. (2005)) is that the simulations that we present follow the dissipationless evolution of DM only. The net effect of dissipation and the condensation of baryons on the anisotropy of satellites is unclear. There are several aspects to consider.

Cosmological gasdynamics simulations of galaxy and cluster formation show that radiative gas cooling results in DM halos that are significantly more spherical in their inner regions than halos in dissipationless simulations (Kazantzidis et al. 2004a). At $\zeta=0.1 R_{180}$, the average increase in the minor-to-major axis ratio is $\Delta(c / a) \sim 0.3$, but this shift is a declining function of $\zeta$, such that at $\zeta \gtrsim 0.5$ it is $\Delta(c / a) \lesssim 0.1$. This does not affect the accretion of satellites along preferred directions (Fig. 6), but will make the halo potential in the inner regions more spherical, reducing any alignment of orbits with the halo major axis. However, this effect is small at large galacto-centric radii and satellites with large apocenters may still move under the influence of an effectively prolate potential.

The presence of a disk could cause the DM halo in its vicinity to adopt a locally-oblate shape aligned with the disk plane (as opposed to the generally prolate shapes seen in cosmological $N$-body simulations), again enhancing destruction of satellites on prograde, co-planar orbits due to anisotropic dynamical friction. If the disk is oriented perpendicular to the halo major axis this would enhance satellite anisotropy compared to our results. Finally, it is possible that the observed, strong anisotropy of the MW satellites is partially due to obscuration by the disk. The incompleteness of the current satellite sample is uncertain, though Willman et al. (2004) argue that a significant fraction of MW satellites at large distances may still be undetected.

\section{SUMMARY AND CONCLUSIONS}

We study the spatial distribution of dwarf subhalos in MWsized DM halos using dissipationless cosmological simulations of the concordance flat $\Lambda \mathrm{CDM}$ cosmology. Specifically, we compare the simulated subhalo populations with the observed distribution of the known MW satellite galaxies. We also test whether the predictions of CDM simulations are consistent with observations using two possible scenarios for mapping luminous satellite galaxies onto subhalos in MW-sized DM halos. In the first scenario, the luminous dwarf satellites are identified using the semi-analytic model for dwarf galaxy formation proposed by Kravtsov et al. (2004). In the second, the luminous satellites reside in those few subhalos with the largest maximum circular velocities $V_{\max }$ (Stoehr et al. 2002, 2003). Our main results can be summarized as follows.

1. The distribution of subhalos in host DM halos is not isotropic. Subhalos are preferentially aligned with the major axis of the triaxial host halo mass distribution. The KS probability of choosing the subhalo populations in our 
simulated MW-sized host halos from an isotropic distribution is $P_{\mathrm{KS}}^{\mathrm{ISO}} \simeq 1.5 \times 10^{-4}$.

2. The method used by Kroupa et al. (2005) does not demonstrate that the MW satellites are inconsistent with either an isotropic underlying distribution or a distribution similar to that of subhalos in CDM simulations. We argue that Kroupa et al. (2005) adopted an incorrect null hypothesis.

3. In terms of angular distribution, the probability for the MW satellites to be drawn from the anisotropic distribution of simulated subhalos is $P_{\mathrm{KS}} \simeq 34 \%$ under the assumption that the MW pole is aligned with the major axis of its host halo. Alternatively, if the MW pole is aligned with the minor axis of the host halo, the KS probability is $P_{\mathrm{KS}} \simeq 2 \%$.

4. The apparent planarity of the MW satellite distribution can be explained by the anisotropy of the subhalo distribution and the relative radial bias of luminous dwarf satellites relative to subhalos. Specifically, the subhalo subsamples that correspond to the luminous subhalos in the model of KGK04 and the eleven highest- $V_{\max }$ subhalos exhibit a degree of planarity that is similar to the observed MW satellites. Note again that in our simulations such planar distributions are likely to be nearly aligned the major axis of the host halo. This, in turn, implies that near alignment of disk pole and halo major axis is required to explain the observed satellite distribution.

5. In agreement with Hartwick (2000), we find that distant $(R \gtrsim 10 \mathrm{kpc})$, metal-poor $([\mathrm{Fe} / \mathrm{H}]<-0.8)$, MW globular clusters exhibit anisotropy similar to that of the dwarf satellites.

6. The observed anisotropy of the MW satellites compared with the CDM predictions for subhalo orientations, along with evidence for the Holmberg effect in other galaxies (Holmberg 1969; Zaritsky et al. 1997; Sales \& Lambas 2004) including the dwarf satellites of M31 (Hartwick 2000), the distribution of MW halo globular clusters, and the indirect arguments of Helmi (2004b), provide evidence for a consistent picture in which the outer DM halos surrounding spiral galaxies should be nearly perpendicular to the disk planes. This has interesting implications for the understanding of disk galaxy formation and the orbital evolution of satellite galaxies.

We discuss the origin of the anisotropy of the subhalo distribution in simulations and show that, similar to galaxy clusters, Galaxy-sized halos accrete substructure along a preferential direction that is strongly correlated with the major axis of the host halo (Fig. 6). We also stress that orbital evolution in a triaxial potential results in an anisotropic spatial distribution of tracer objects, even if their accretion is isotropic.

The fact that consistency of observations with the $\Lambda \mathrm{CDM}$ prediction appears to require near alignment of disk angular momenta with the major axis of host halos is surprising. The angular momenta of halos are typically aligned with their minor axes (e.g., Warren et al. 1992; Porciani et al. 2002; Faltenbacher et al. 2005). This could indicate that disk formation is accompanied by the evolution of the angular momentum of the baryonic material as it cools and condenses in the center. This would not be surprising given that condensation of baryons has a significant impact on the density and shape of the surrounding DM halo (Gnedin et al. 2004; Kazantzidis et al. 2004a). However, more detailed numerical studies of disk galaxy formation are needed to understand the underlying processes in detail.

It would be interesting to test whether the putative disk-halo configuration is ubiquitous. Such tests can be attempted using several approaches. For example, a particular alignment hypothesis can be tested with weak lensing measurements. Another statistical test can be done using the projected distributions of satellite galaxies in large galaxy surveys. In the Appendix, we present the distribution of expected satellite angles with respect to the major axis of the projected host halo ellipse (a more complete study will be presented in a forthcoming paper, Zentner et al., in preparation). This predicted distribution can be compared to the observed satellite distribution around the major axis of the light distribution of disk galaxies. Such a comparison can test any correlation between disk orientation and dark matter elongation. A detection would indirectly indicate a preferential orientation of disk galaxies in DM halos, while a null result would have the interesting implication that the correlation between disk and halo orientation is, at most, weak. These tests should be feasible with existing large galaxy surveys, such as the Sloan Digital Sky Survey. We therefore expect progress in this direction in the near future.

We would like to thank Michael Blanton, James Bullock, Q. Y. Gong, Amina Helmi, David Hogg, Savvas Koushiappas, Erin Sheldon, Monica Valluri, Risa Wechsler, and Beth Willman for useful discussions. We are grateful to Frank van den Bosch, Stelios Kazantzidis, Alexander Knebe, and James Taylor for detailed comments on an earlier draft of this manuscript and several helpful suggestions. We thank Tad Pryor for sharing recent results on the orbital planes of the MW satellites. We acknowledge use of the publicly-available MW Globular Cluster Catalog compiled by William Harris. ARZ is supported by The Kavli Institute for Cosmological Physics (KICP) at The University of Chicago and The National Science Foundation (NSF) under grant NSF PHY 0114422. AVK is supported by the NSF under grants No. AST-0206216 and AST-0239759, by NASA through grant NAG5-13274, and by the KICP at the University of Chicago. OYG is funded by NASA ATP grant NNG04GK68G. AAK is supported by the NSF grant AST0206216 to NMSU. The simulations presented here were performed on the Origin2000 at the National Center for Supercomputing Applications (Urbana-Champaign, Illinois, USA). This research made use of the NASA Astrophysics Data System.

\section{APPENDIX}

\section{SATELLITE ANISOTROPY IN PROJECTION}

Above, we demonstrated that satellite halos are not distributed isotropically about their main host halos, but are preferentially aligned along the major axes of their hosts. This, coupled with the fact that the luminous dwarfs likely form in a biased subset of DM subhalos, greatly mitigates any claims that the CDM paradigm of structure formation is in conflict with the observed MW satellites. Agreement seems to require that the rotation axes of disk galaxies are nearly aligned with the major axes of their host halos. It would be interesting to examine populations of satellites around other galaxies and to compare them to theoretical predictions. This requires examining the angular distributions of satellites in two-dimensional (2D) projection, 
where the anisotropy is somewhat diluted. We present the projected distribution of subhalos relative to their host halos in this Appendix.

To determine the projected subhalo angular distributions, we take three orthogonal projections of each host halo and find the principal axes of the $2 D$ mass distributions by diagonalizing the $2 D$ analog of the moment of inertia tensor [Eq. (1)]. For each satellite, we determine the angle $\phi$, between the major axis of inertia and the satellite position. In practice, correlated galaxies along the line-of-sight to the host galaxy contribute to the differential angular distribution as it is difficult to remove objects at a fixed three-dimensional distance. To account approximately for this, we include in our sample all halos and subhalos within a three-dimensional distance $r_{\text {cut }}=2 R_{180}$ from the host halo center, and within a projected, $2 D$ distance $r_{\mathrm{p}}=300 h^{-1} \mathrm{kpc}$. It is not possible to extend our projection to larger three-dimensional radius because $2 R_{180}$ marks the maximum extent of the highresolution regions of the simulation (see $\S 2.1$ ). However, in a forthcoming study we examine the angular distributions of halos relative to nearby hosts in projection and find that the differential distributions are not strongly affected by extending the projection region (Zentner et al., in preparation).

Figure A9 shows differential distributions of $\phi$ for the satellites of our simulated halos. The anisotropy in $2 D$ projection remains clear. The probability that a satellite lies at the major axis $\left(\phi \leq 10^{\circ}\right)$ of the ellipse is $\gtrsim 50 \%$ larger than the probability that it lies near the minor axis $\left(\phi \geq 80^{\circ}\right)$. Additionally, the degree of anisotropy appears to be weakly dependent on subhalo size over the relatively small range of subhalo $V_{\max }$ that we can reliably probe.

The coordinate system used in any observational study will be defined by the distribution of light rather than by the unseen distribution of DM. As such, Fig. A9 requires a strong correlation between the orientation of the luminous component of the host galaxy and the principal axes of its host halo. In the absence of such a correlation, any anisotropy would be diluted away in a study of many host systems. This fact provides an interesting constraint on theories of galaxy formation, as it can potentially serve to provide some statistical measure of the orientations of disk and/or elliptical galaxies within their host halos.

\section{REFERENCES}

Abadi, M. G., Navarro, J. F., Steinmetz, M., \& Eke, V. R. 2003, ApJ, 597, 21

Armandroff, T. E. 1989, AJ, 97, 375

Barnes, J. A. \& Efstathiou, G. 1987, ApJ, 319, 575

Barnes, J. E. \& Hernquist, L. 1996, ApJ, 471, 115

Benson, A. J., Lacey, C. G., Frenk, C. S., Baugh, C. M., \& Cole, S. 2004, MNRAS, 351, 1215

Binney, J. J. 1977, MNRAS, 181, 735

Blumenthal, G. R., Faber, S. M., Primack, J. R., \& Rees, M. J. 1984, Nature, 311,517

Bond, J. R., Kofmann, L., \& Pogosyan, D. 1996, Nature, 380, 603

Brainerd, T. G. 2004, ApJL, Submitted (astro-ph/0408559)

Bullock, J. S. 2002, in in Proceedings of the Yale Cosmology Workshop "The Shapes of Galaxies and Their Dark Matter Halos", (28-30 May, 2001). P.Natarajan (ed.). Singapore: World Scientific, 109

Bullock, J. S., Kolatt, T. S., Sigad, Y., Somerville, R. S., Kravtsov, A. V., Klypin, A. A., Primack, J. R., \& Dekel, A. 2001, MNRAS, 321, 559

Bullock, J. S., Kravtsov, A. V., \& Weinberg, D. H. 2000, ApJ, 539, 517

Chen, D. N., Jing, Y. P., \& Yoshikaw, K. 2003, ApJ, 597, 35

Colberg, J. M., Krughoff, K. S., \& Connolly, A. J. 2004, MNRAS in press (astro-ph/0406665)

Colín, P., Klypin, A. A., Kravtsov, A. V., \& Khokhlov, A. M. 1999, ApJ, 523, 32

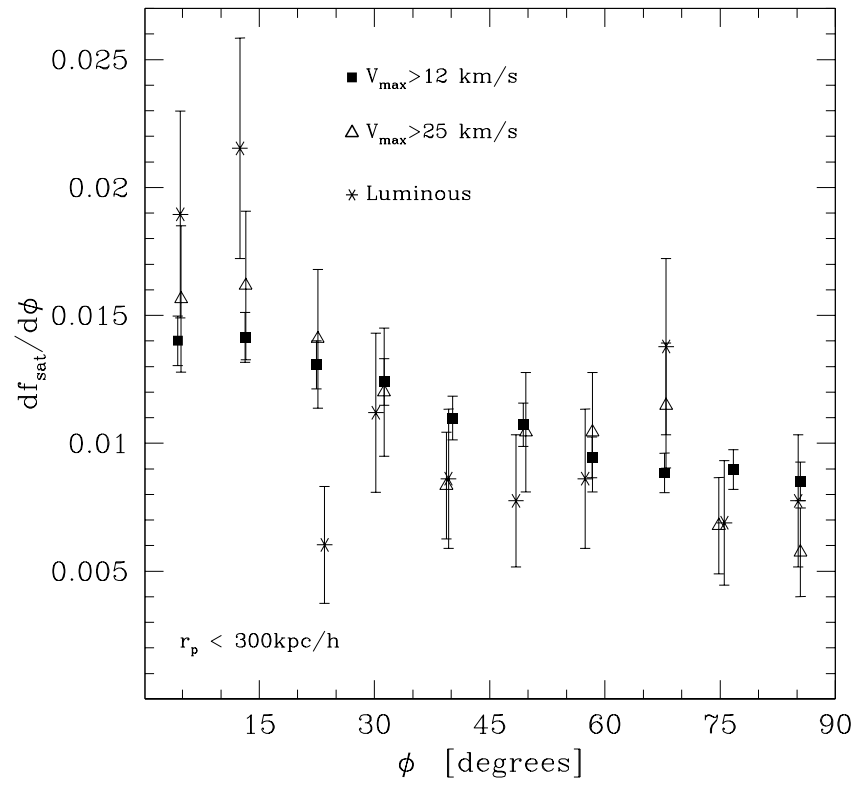

FIG. A9.- The distribution of subhalo angular position with respect to the long axis of the host halo in $2 D$ projection. The fractional distribution of subhalos $\mathrm{d} f_{\text {sat }} / \mathrm{d} \phi$, was computed by summing over three orthogonal projections for each host halo. We show the distribution for all halos and subhalos within a three-dimensional distance $r_{\text {cut }} \leq 2 R_{180}$ and a projected distance of $r_{\mathrm{p}} \leq 300 \mathrm{~h}^{-1} \mathrm{kpc}$ of the center of the host halo. The filled squares represent all subhalos with $V_{\max } \geq 12 \mathrm{~km} \mathrm{~s}^{-1}$. The open triangles represent all subhalos with $V_{\max } \geq 25 \mathrm{~km} \mathrm{~s}^{-1}$. The stars represent the distribution of satellites that should host luminous galaxies according to the model of Kravtsov et al. (2004)

Colless, M., Dalton, G., Maddox, S., Sutherland, W., \& the $2 \mathrm{dF}$ collaboration. 2001, MNRAS, 328, 1039

De Lucia, G., Kauffmann, G., Springel, V., White, S. D. M., Lanzoni, B., Stoehr, F., Tormen, G., \& Yoshida, N. 2004, MNRAS, 348, 333

Diemand, J., Moore, B., \& Stadel, J. 2004, MNRAS, 352, 535

Doroshkevich, A. G. 1970, Astrofizika, 6, 581

Dubinski, J. 1994, ApJ, 431, 617

Dubinski, J. \& Carlberg, R. G. 1991, ApJ, 378, 496

Efstathiou, G. \& Jones, B. J. T. 1979, MNRAS, 186, 133

Fall, S. M. \& Efstathiou, G. 1980, MNRAS, 193, 189

Faltenbacher, A., Allgood, B., Gottloeber, S., Yepes, G., \& Hoffman, Y. 2005, MNRAS submitted (astro-ph/0501452)

Font, A. S., Navarro, J. F., Stadel, J., \& Quinn, T. 2001, ApJ, 563, L1

Frenk, C. S. \& White, S. D. M. 1982, MNRAS, 198, 173

Gao, L., De Lucia, G., White, S. D. M., \& Jenkins, A. 2004a, MNRAS, 352, L1

Gao, L., White, S. D. M., Jenkins, A., Stoehr, F., \& Springel, V. 2004b, MNRAS submitted (astro-ph/0404589)

Gerhard, O. E. \& Binney, J. J. 1985, MNRAS, 216, 467

Ghigna, S., Moore, B., Governato, F., Lake, G., Quinn, T., \& Stadel, J. 2000, ApJ, 544, 616

Ghigna, S., Moore, B., Governato, F. \& Stadel, J. 1998, MNRAS, 300, 146

Gnedin, O. Y., Kravtsov, A. V., Klypin, A. A., \& Nagai, D. 2004, ApJ, 616, 16

Grebel, E. K., Gallagher, J. S., \& Harbeck, D. 2003, AJ, 125, 1926

Grebel, E. K., Kolatt, T., \& Brandner, W. 1999, in IAU Symposium, 447

Harris, W. E. 1996, AJ, 112, 1487

Hartwick, F. D. A. 1996, in ASP Conf. Ser. 92, Formation of the Galactic Halo...Inside and Out, 444

Hartwick, F. D. A. 2000, AJ, 119, 2248

Hawley, D. L. \& Peebles, P. J. E. 1975, AJ, 80, 477

Hayashi, D., Navarro, J. F., Taylor, J. E., Stadel, J., \& Quinn, T. 2003, ApJ, 584,541

Helmi, A. 2004a, MNRAS, 351, 643

-. 2004b, ApJ, 610, L97

Helmi, A. \& White, S. D. M. 1999, MNRAS, 307, 495

Hoekstra, H., Yee, H. K. C., \& Gladders, M. D. 2004, ApJ, 606, 67

Holmberg, E. 1969, Arkiv Astron., 5, 305

Huang, S. \& Carlberg, R. G. 1997, ApJ, 480, 503

Ibata, R., Lewis, G. F., Irwin, M., Totten, E., \& Quinn, T. 2001, ApJ, 551, 294

Ibata, R. A. \& Razoumov, A. O. 1998, Astron. \& Astrophys., 336, 130 
Jing, Y. P. \& Suto, Y. 2002, ApJ, 574, 538

Johnston, K. V., Law, D. R., \& Majewski, S. R. 2004, ApJ submitted (astro$\mathrm{ph} / 0407565)$

Kang, X., Mao, S., Gao, L., \& Jing, Y. P. 2005, Astron. \& Astrophys. Submitted (astro-ph/0501333)

Kauffmann, G., White, S. D. M., \& Guiderdoni, B. 1993, MNRAS, 264, 201

Kazantzidis, S., Kravtsov, A. V., Zentner, A. R., Allgood, B. A., Nagai, D., \& Moore, B. 2004a, ApJL, 611, L73

Kazantzidis, S., Mayer, L., Mastropietro, C., Diemand, J., Stadel, J., \& Moore, B. 2004b, ApJ, 608, 663

Klypin, A. A., Gottlöber, S., Kravtsov, A. V., \& Khokhlov, A. M. 1999b, ApJ, 516,530

Klypin, A. A., Kravtsov, A. V., Bullock, J. S., \& Primack, J. R. 2001, ApJ, 554, 903

Klypin, A. A., Kravtsov, A. V., Valenzuela, O., \& Prada, F. 1999a, ApJ, 522, 82

Klypin, A. A. \& Shandarin, S. F. 1983, MNRAS, 204, 891

Knebe, A., Gill, S. P. D., Gibson, B. K., Lewis, G. F., Ibata, R. A., \& Dopita, M. A. 2004, ApJ, 603, 7

Kravtsov, A. V. 1999, PhD thesis, New Mexico State University

Kravtsov, A. V., Gnedin, O. Y., \& Klypin, A. A. 2004, ApJ, 609, 482 (KGK04)

Kravtsov, A. V., Klypin, A. A., \& Khokhlov, A. M. 1997, ApJS, 111, 73

Kroupa, P., Theis, C., \& Boily, C. M. 2005, Astron. \& Astrophys., 431, 517

Law, D. R., Majewski, S. R., Johnston, K. V., \& Strutskie, M. F. 2004, in Satellites and Tidal Streams

Lynden-Bell, D. 1982, Obs., 102, 202

MacGillivray, H. T., Dodd, R. J., McNally, B. V., \& Corwin, H. G. 1982, MNRAS, 198, 605

Majewski, S. R. 1994, ApJL, 431, L17

Majewski, S. R., Kunkel, W. E., Law, D. R., Patterson, R. J., Polak, A. A., Rocha-Pinto, H. J., Crane, J. D., Frinchaboy, P. M., Hummels, C. B., Johnston, K. V., Rhee, J., Skrutskie, M. F., \& Weinberg, M. 2004, AJ, 128, 245

Majewski, S. R., Skrutskie, M. F., Weinberg, M. D., \& Ostheimer, J. C. 2003, ApJ, 599, 1082

Martínez-Delgado, D., Gómez-Flechoso, M. Á., Aparicio, A., \& Carrera, R. 2004, ApJ, 601, 242

Mateo, M. L. 1998, ARA\&A, 36, 435

Merritt, D. \& Quinlan, G. D. 1998, ApJ, 498, 625

Moore, B., Ghigna, S., Governato, F., Lake, G., Quinn, T., Stadel, J., \& Tozzi, P. 1999, ApJL, 524, L9

Nagai, D. \& Kravtsov, A. V. 2005, ApJ, 618, 557

Navarro, J. F., Abadi, M. G., \& Steinmetz, M. 2004, ApJ, 613, L41

Navarro, J. F., Frenk, C. S., \& White, S. D. M. 1994, MNRAS, 267, L1

-. 1995, MNRAS, 275, 56

-. 1997, ApJ, 490, 493 (NFW)

Peñarrubia, J., Kroupa, P., \& Boily, C. M. 2002, MNRAS, 333, 779

Peebles, P. J. E. 1969, ApJ, 155, 393

Piatek, S., Pryor, C., Bristow, P., Olszewski, E. W., Harris, H. C., Mateo, M., Minniti, D., \& Tinney, C. G. 2005, AJ, Submitted

Porciani, C., Dekel, A., \& Hoffmann, Y. 2002, MNRAS, 332, 339

Quinn, P. J. \& Goodman, J. 1986, ApJ, 309, 472

Reed, D., Gardner, J., Quinn, T., Stadel, J., Fardal, M., Lake, G., \& Governato, F. 2003, MNRAS, 346, 565

Reed, D., Governato, F., Quinn, T., Gardner, J., Stadel, J., \& Lake, G. 2004, MNRAS submitted (astro-ph/0406034)

Ricotti, M. \& Gnedin, N. Y. 2004, ApJ submitted (astro-ph/0408563)

Sales, L. \& Lambas, D. G. 2004, MNRAS, 348, 1236

Sharp, N. A., Lin, D. N. C., \& White, S. D. M. 1979, MNRAS, 187, 287

Springel, V., White, S. D. M., Tormen, G., \& Kauffmann, G. 2001, MNRAS, 328,726

Statler, T. S. 1987, ApJ, 321, 113

Stoehr, F., White, S. D. M., Springel, V., Tormen, G., \& Yoshida, N. 2003, MNRAS, 345, 1313

Stoehr, F., White, S. D. M., Tormen, G., \& Springel, V. 2002, MNRAS, 335, L84

Strauss, M. A., Weinberg, D. H., Lupton, R. H., \& the SDSS collaboration. 2002, AJ, 124, 1810

Taylor, J. E. \& Babul, A. 2001, ApJ, 559, 716

-. 2004, MNRAS, 348, 811

Taylor, J. E., Silk, J., \& Babul, A. 2003, in IAU Symp. 220: Dark Matter in Galaxies, 118

Tormen, G. 1997, MNRAS, 290, 211

Tóth, G. \& Ostriker, J. P. 1992, ApJ, 389, 5

Udry, S. \& Martinet, L. 1994, Astron. \& Astrophys., 281, 314

Valluri, M. \& Merritt, D. 1998, ApJ, 506, 686

van den Bosch, F. C., Abel, T., Croft, R. A. C., Hernquist, L., \& White, S. D. M. 2002, ApJ, 576, 21

Velázquez, H. \& White, S. D. M. 1999, MNRAS, 304, 254

Warren, M. S., Quinn, P. J., Salmon, J. K., \& Zurek, W. H. 1992, ApJ, 340, 771

West, M. J. \& Blakeslee, J. P. 2000, ApJL, 543, L27

Willman, B., Governato, F., Dalcanton, J. J., Reed, D., \& Quinn, T. 2004, MNRAS, 353, 639

York, D. G., Adelman, J., Anderson, J. E., Anderson, S. F., Annis, J., \& the SDSS collaboration. 2000, AJ, 120, 1579

Zaritsky, D. \& Gonzalez, A. H. 1999, PASP, 111, 1508
Zaritsky, D., Smith, R., Frenk, C. S., \& White, S. D. M. 1997, ApJL, 478, L53 Zaritsky, D. \& White, S. D. M. 1994, ApJ, 435, 599

Zentner, A. R., Berlind, A. A., Bullock, J. S., Kravtsov, A. V., \& Wechsler, R. H. 2005, ApJ, In Press (astro-ph/0411586), 624

Zentner, A. R. \& Bullock, J. S. 2003, ApJ, 598, 49

Zinn, R. 1985, ApJ, 293, 424 